This is a preprint of: "Global phase portraits of some reversible cubic centers with noncollinear singularities", Magdalena Caubergh, Joan Torregrosa, Internat. J. Bifur. Chaos Appl. Sci. Engrg., vol. 23(9), 1350161 (30 pages), 2013.

DOI: [10.1142/S0218127413501617]

\title{
GLOBAL PHASE PORTRAITS OF SOME REVERSIBLE CUBIC CENTERS WITH NONCOLLINEAR SINGULARITIES
}

\author{
M. CAUBERGH AND J. TORREGROSA
}

\begin{abstract}
The results in this paper show that the cubic vector fields $\dot{x}=-y+$ $M(x, y)-y\left(x^{2}+y^{2}\right), \dot{y}=x+N(x, y)+x\left(x^{2}+y^{2}\right)$, where $M, N$ are quadratic homogeneous polynomials, having simultaneously a center at the origin and at infinity, have at least 61 and at most 68 topologically different phase portraits. To this end the reversible subfamily defined by $M(x, y)=-\gamma x y, N(x, y)=(\gamma-\lambda) x^{2}+\alpha^{2} \lambda y^{2}$ with $\alpha, \gamma \in \mathbb{R}$ and $\lambda \neq 0$, is studied in detail and it is shown to have at least 48 and at most 55 topologically different phase portraits. In particular, there are exactly 5 for $\gamma \lambda<0$ and at least 46 for $\gamma \lambda>0$. Furthermore, the global bifurcation diagram is analyzed.
\end{abstract}

\section{INTRODUCTION}

This paper finishes the topological classification of the global phase portraits on the Poincaré disc of the 6-parameter family of cubic differential equations

$$
\begin{aligned}
& \dot{x}=-y+a x^{2}+b x y+c y^{2}-y\left(x^{2}+y^{2}\right), \\
& \dot{y}=x+e x^{2}+f x y+g y^{2}+x\left(x^{2}+y^{2}\right),
\end{aligned}
$$

that have simultaneously a center in the origin and at infinity for $a, b, c, e, f, g \in \mathbb{R}$. This study is started in $[8,9]$.

Recently related analysis are done in $[3,4,5,7,11,12]$. In particular, in [12], the so-called SIS-model is considered, that is used in the study of infectious diseases. The papers $[3,4,5,7,12]$ contribute to the classification of planar quadratic differential systems; due to the 6-dimensional parameter and the richness of phase portraits, its bifurcation diagram also is studied for intrinsic subclasses reducing its dimension, and similarly these sub-bifurcation diagrams then are analyzed by slicing and imbedding in projective planes. Furthermore another generalization can be found in [11], where a global topological classification is studied for a 1-parameter cubic Hamiltonian planar differential system in which a finite center is linked to singularities at infinity.

In [6] the differential systems (1) are characterized by a Hamiltonian class and a reversible class, that is symmetric with respective to straight lines. In [8] and [9] respectively the classification is obtained for the full Hamiltonian class and part of the reversible class, i.e the ones having infinitely many singularities or all singularities on the line of symmetry. The classification of the remaining vector fields of (1) is subject

2010 Mathematics Subject Classification. Primary 34C23; Secondary 34C25, 34C37, 37G10, 37C27, $34 \mathrm{C} 29,34 \mathrm{C} 05$.

Key words and phrases. reversible planar vector fields, cubic vector fields, global classification of phase portraits, bifurcation diagram.

Partially supported by the MINECO/FEDER grant number MTM2008-03437 and by the AGAUR grant number 2009SGR 410. The first author also is supported by the Ramón y Cajal grant number RYC-2011-07730. 
of this paper. These can be represented by the 3-parameter family $Y_{(\alpha, \gamma, \lambda)}$ given by

$$
\begin{aligned}
& \dot{x}=-y-\gamma x y-y\left(x^{2}+y^{2}\right), \\
& \dot{y}=x+(\gamma-\lambda) x^{2}+\alpha^{2} \lambda y^{2}+x\left(x^{2}+y^{2}\right),
\end{aligned}
$$

where $\alpha, \gamma, \lambda \in \mathbb{R}$ and $\lambda \neq 0$. These vector fields are reversible because they are invariant with respect to the transformation $(x, y, t, \alpha, \gamma, \lambda) \mapsto(x,-y,-t, \alpha, \gamma, \lambda)$ and hence their phase portraits are symmetric with respect to the horizontal axis when reversing time. It is found in Section 2 that the analysis can be restricted to the semi-algebraic set

$$
\mathcal{P}=\left\{(\alpha, \gamma, \lambda) \in \mathbb{R}^{3}: \alpha>0, \gamma>0, \lambda \neq 0\right\} .
$$

The systematic classification here obtained for (2) with respect to the number of singularities is summarized in the following theorem. Recall that isolated periodic orbits are so-called limit cycles.

Theorem 1. The subfamily of cubic reversible vector fields $Y_{(\alpha, \gamma, \lambda)}$, given in (2) with $\alpha, \gamma, \lambda \in \mathbb{R}$ and $\lambda \neq 0$, has at most 55 topologically different global phase portraits of which at least 48 are realized, and that are drawn in Figures 1, 15, 16, 17, 20, 21, 22, 24, 25 and 28. More precisely, if the lower bound resp. upper bound for the number of topologically different phase portraits having $i$ singularities is denoted by $L_{i}$ resp. $U_{i}, i \in \mathbb{N}$, then $\sum_{i=1}^{7} L_{i}=48$ and $\sum_{i=1}^{7} U_{i}=55$, where $L=(1,1,4,3,10,8,21)$ and $U=(1,1,4,6,13,9,21)$. In particular, the number $n$ of singularities is finite with $n \in\{1,2, \ldots, 7\}$ and there are no limit cycles.

Notice that the figures mentioned in previous theorem only show the phase portraits that constitute the lower bound. Furthermore these phase portraits are grouped first according to the number of singularities and second according to local bifurcation regions. Moreover the specific order and composition of the phase portraits respects the subsequent global bifurcation phenomena that happen between adjacent phase portraits and is detailed for each case in the corresponding section. This also is the reason why some topologically equivalent phase portraits appear.

Adding results from [9] to Theorem 1 to deal with the case $\lambda=0$ leads to the classification of the full 3-parameter family $Y_{(\alpha, \gamma, \lambda)}$, by adding one more phase portrait that has infinitely many singularities.

Corollary 2. The cubic reversible vector fields $Y_{(\alpha, \gamma, \lambda)}$, given in (2) with $\alpha, \gamma, \lambda \in \mathbb{R}$, has at most 56 topologically different global phase portraits of which at least 49 are realized. In particular, the number $n$ of singularities is finite if and only if $\lambda \neq 0$ or $\lambda=0, \gamma \leq 2$. Furthermore, none of these phase portraits has limit cycles.

Denote by $\mathcal{F}$ the quotient space of all phase portraits of (1) with respect to topological equivalence. Consider the subsets $\mathcal{F}_{H} \subset \mathcal{F}$ and $\mathcal{F}_{R} \subset \mathcal{F}$ that correspond to the phase portraits of the Hamiltonian and reversible class of (1) respectively. Write $|\mathcal{S}|$ to denote the cardinal number of $\mathcal{S}$, and hence corresponding to the topologically different phase portraits. Then, combining Theorem 1 and the results from [8, 9] leads to the following classification for the full family (1).

Corollary 3. The 6-parameter family (1) having simultaneously a center at the origin and at infinity induces $61 \leq|\mathcal{F}| \leq 68$ topologically different phase portraits. In particular, $\left|\mathcal{F}_{H}\right|=22,53 \leq\left|\mathcal{F}_{R}\right| \leq 60$ and $\left|\mathcal{F}_{H} \cap \mathcal{F}_{R}\right|=14$. Furthermore, none of these phase portraits present limit cycles and phase portraits in $\mathcal{S}$ have maximally $n_{P A}(\mathcal{S})$ 
disjoint period annuli, where $n_{P A}(\mathcal{F})=n_{P A}\left(\mathcal{F}_{H}\right)=7, n_{P A}\left(\mathcal{F}_{R}\right)=n_{P A}\left(\mathcal{F}_{H} \cap \mathcal{F}_{R}\right)=6$ and $n_{P A}\left(\mathcal{F}_{R} \backslash \mathcal{F}_{H}\right)=4$.

In studying the topologically different global phase portraits a great role is played by the bifurcation diagram of the global phase portraits in terms of the parameter $(\alpha, \gamma, \lambda) \in \mathcal{P}$. To simplify the reading we use, whenever no confusion is possible, the notation $\zeta=(\alpha, \gamma, \lambda)$.

Throughout this paper we shortly speak of the local (resp. global) bifurcation diagram referring to the bifurcation diagram of the local (resp. global) phase portraits with respect to topological equivalence in terms of the parameter $\zeta$. In this, local means near the singularities. As such local (resp. global) bifurcation surfaces and curves are the bifurcation surfaces and curves dealing with local (resp. global) bifurcation phenomena. First, in Section 3, we study the local bifurcation diagram. Next the global bifurcation diagram is obtained by adding global bifurcation surfaces to it, that are determined by crossing of separatrices. In the transition from $\lambda<0$ to $\lambda>0$ the global center of the harmonic oscillator is disturbed by a circle of singularities at $1+\gamma x+x^{2}+y^{2}=0$ (see [9] and Proposition 29). Due to their different nature, the cases $\lambda<0$ and $\lambda>0$ are treated separately. To study this 3 -dimensional bifurcation diagram systematically we consider slices of $\mathcal{P}$ for fixed $\lambda$, that we denote by $\mathcal{P}_{\lambda}=\{\zeta: \alpha, \gamma>0\}$. Then characteristic slices of the global bifurcation diagram in $\mathcal{P}_{\lambda}$ are studied in Sections 6 and 7 for $\lambda<0$ and $\lambda>0$ respectively.

With increasing $\lambda$ subsequently more bifurcation surfaces are encountered. Furthermore, opposed to the case $\lambda>0$, in case $\lambda<0$ there are no Hamiltonian phase portraits and the local bifurcation phenomena determine the global ones. This is illustrated by the bifurcation diagrams in Figure 2 for $\lambda<0$ and in Figures 7, 32 and 33 for $\lambda>0$. In Section 3 it is seen that the local bifurcation diagram is determined by the following semi-algebraic subsets of $\mathcal{P}=\mathcal{P}_{-} \cup \mathcal{P}_{+}$, where $\mathcal{P}_{-}=\mathcal{P} \cap\{\lambda<0\}$ and $\mathcal{P}_{+}=\mathcal{P} \cap\{\lambda>0\}$ :

$$
\begin{aligned}
\mathcal{F}_{u} & =\{\gamma>\lambda+2\}, & \mathcal{E}_{u} & =\{\gamma>\lambda-2\}, \\
\mathcal{F} & =\{\gamma=\lambda+2\}, & \mathcal{E} & =\{\gamma=\lambda-2\}, \\
\mathcal{F}_{d} & =\{\gamma<\lambda+2\}, & \mathcal{E}_{d} & =\{\gamma<\lambda-2\}, \\
\mathcal{G}_{l} & =\left\{\alpha^{2} \gamma^{2}-4\left(\alpha^{2}+1\right)<0\right\}, & \mathcal{H}_{l} & =\left\{2 \alpha^{2} \lambda-\gamma<0\right\}, \\
\mathcal{G} & =\left\{\alpha^{2} \gamma^{2}-4\left(\alpha^{2}+1\right)=0\right\}, & \mathcal{H} & =\left\{2 \alpha^{2} \lambda-\gamma=0\right\}, \\
\mathcal{G}_{r} & =\left\{\alpha^{2} \gamma^{2}-4\left(\alpha^{2}+1\right)>0\right\}, & \mathcal{H}_{r} & =\left\{2 \alpha^{2} \lambda-\gamma>0\right\} .
\end{aligned}
$$

Furthermore we use the notation $\mathcal{E}_{\lambda}, \mathcal{F}_{\lambda}$ and $\mathcal{H}_{\lambda}$ for the slices obtained by intersecting the corresponding bifurcation surfaces with $\mathcal{P}_{\lambda}$ for fixed $\lambda \neq 0$. In Lemma 6 it is found that the Hamiltonian reversible vector fields $Y_{\zeta}$ correspond with the parameter $\zeta \in \mathcal{H}$. A characteristic slice of $\mathcal{H}_{\lambda}$ is non-empty only for $\lambda>0$ and its projection in the $(\alpha, \gamma)$-plane is presented in Figure 6 . The global bifurcation diagram restricted to $\mathcal{H}$ is detailed in Section 5 using results from [8]; in particular it is shown in Figure 9 and the corresponding phase portraits are shown in Figure 8.

Using the subsets introduced in (4) we now state the main results of this paper, distinguishing between $\lambda<0$ (i.e., in $\mathcal{P}_{-}$) and $\lambda>0$ (i.e., in $\mathcal{P}_{+}$).

Theorem 4. The family (2) restricted to $\mathcal{P}_{-}$has exactly 5 topologically different global phase portraits, that are drawn in Figure 1. Furthermore the global phase portrait of $Y_{\zeta}$ is uniquely determined by the number $n$ of singularities; in particular, up to topological 
equivalence, the phase portrait is equal to $\bar{n}$ with $n \in\{1,2,3,5,7\}$. For fixed $\lambda<0$ characteristic slices of the global bifurcation diagram are shown in Figure 2.

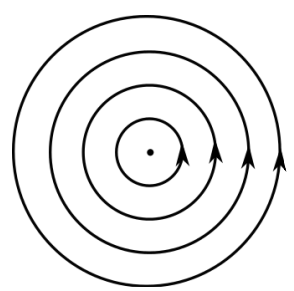

$\overline{1}$

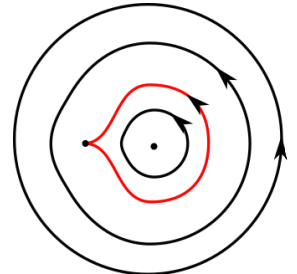

$\overline{2}$

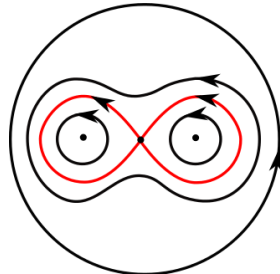

$\overline{3}$

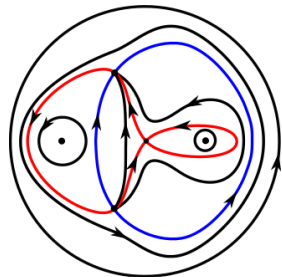

$\overline{5}$

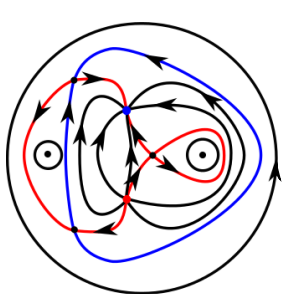

$\overline{7}$

Figure 1. Phase portraits of $(2)$ in $\mathcal{P}_{-}$, see Theorem 4.
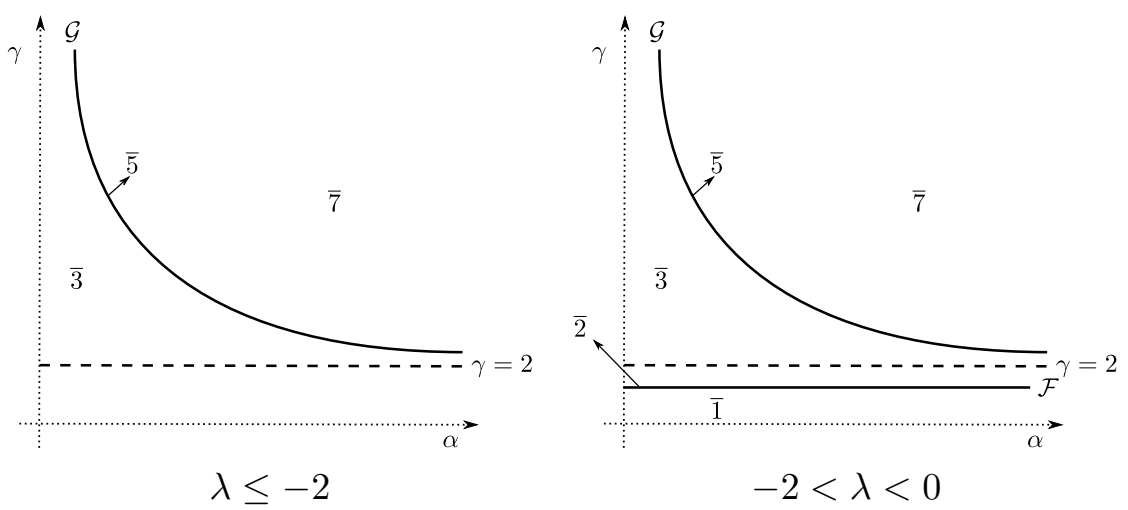

FiguRE 2. Characteristic slices of the local and global bifurcation diagram for $(2)$ in $\mathcal{P}_{\lambda}$ for fixed $\lambda<0$ (see also Figure 1 ).

Theorem 5. The family (2) restricted to $\mathcal{P}_{+}$has at most 53 topologically different global phase portraits of which at least 46 are realized, and that are drawn in Figures 15, 16, 17, 20, 21, 22, 24, 25 and 28. More precisely, if the lower bound resp. upper bound for the number of topologically different phase portraits having $i$ singularities is denoted by $L_{i}^{+}$ resp. $U_{i}^{+}, i \in \mathbb{N}$, then $\sum_{i=1}^{7} L_{i}^{+}=46$ and $\sum_{i=1}^{7} U_{i}^{+}=53$, where $L^{+}=(1,1,4,3,9,8,20)$ and $U^{+}=(1,1,4,6,12,9,20)$.

For $\lambda>0$ the bifurcation diagram as well as the notation for the global phase portraits is more involved than for $\lambda<0$. Typically the notation then is of the form $n_{q}^{p}$ where $n$ refers to the total number of singularities and the symbols that appear in the indices $p$ and $q$ different from $0,+,-, 1$, * refer to the local bifurcation phenomena (i.e. appearance and disappearance of the singularities and their local behavior). The full notation is explained in Section 3.2 related to the local bifurcations and in Section 7 related to the global ones.

The paper is organized as follows. The parameter space is reduced to $\mathcal{P}$ in Section 2 and the local bifurcation diagram is analyzed in Section 3. In Section 4 it is shown that (2) has no limit cycles and conditions for homoclinic or heteroclinic orbits to exist are found. In Sections 5, 6 and 7 we systematically study the global bifurcation diagram for (2) restricted to $\mathcal{P} \cap \mathcal{H}, \mathcal{P}_{-}$and $\mathcal{P}_{+}$respectively. In Section 5 the global 
bifurcation diagram for the Hamiltonian reversible subfamily is obtained. Notice that we already dealt with this problem in [8] presenting the topological classification of the phase portraits but not the bifurcation diagram. Here we recall this result and rewrite it in terms of $\zeta$ in Theorem 17, adding the bifurcation diagram in Figure 9. In particular unfolding the Hamiltonian vector fields in (2) is one of the key tools used to prove the existence of nearby global phase portraits of (2) for $\lambda>0$. Next in Sections 6 and 7 the cases $\lambda<0$ and $\lambda>0$ are dealt with respectively and in particular we prove Theorems 4 and 5 . The case $\lambda>0$ is dealt with systematically depending on the number of singularities that are present. First we obtain rather directly the classification for the cases of one, two and three singularities, that is stated in Proposition 19. Due to increasing complexity the cases of seven, five, six and four singularities are subsequently dealt with in Sections 7.2, 7.3, 7.4 and 7.5. Section 7.2 treats the case when the number of singularities is maximal, independently analyzing all possible global phase portraits, which are listed in Proposition 20. Then the other cases each time are considered as bifurcation of previously treated cases and the key in proving the existence of possible phase portraits is based on arguments of continuity. This is the reason why first the cases of seven and five singularities are studied and then subsequently the cases of six and four singularities. Finally in Section 8 we combine Theorems 4 and 5 and extend them to the boundary of $\mathcal{P}_{ \pm}$using results from $[8,9]$. In this way, results for the full parameter space are obtained that prove Theorem 1 and Corollaries 2 and 3.

The study is mainly done using classical techniques in qualitative theory of differential equations in the plane (see $[2,10]$ ). To be sure to cover all global phase portraits, the parameter space is examined systematically by the possible bifurcations. Although some of these bifurcations result in topologically equivalent differential systems, the corresponding phase portraits are explicitly drawn to stress on the bifurcation phenomena. Besides local also global bifurcations take place. In particular a rigorous study is done of how the separatrices starting from singularities mutually cross and bifurcation methods are used to analyze near-Hamiltonian cases.

We end this introduction by exposing part of the complexity of the problem considered in this paper. From the quantitative part of view, the number of topologically different phase portraits is surprisingly small, compared to the number that one obtains by combinatorial computation after a mere study near the singularities. For instance considering phase portraits having seven singularities there are 8 attracting and 8 repelling separatrices that can connect. Computing their possibilities to connect one finds $8 !=40320$ different phase portraits. Using the reversibility property and a concise analysis of the $\alpha$ - and $\omega$-limit sets of the separatrices of the saddle points reduces this number to exactly 1 for $\mathcal{P}_{-}$and 20 for $\mathcal{P}_{+}$. The total number of possible topologically different phase portraits is reduced to 5 for $\mathcal{P}_{-}$and at most 53 for $\mathcal{P}_{+}$. Adding, in the case of $\mathcal{P}_{+}$, a careful numerical study of the relative positions of the separatrices in case of four singularities this total number further reduces to 50. Only 46 of these 50 phase portraits are traced when for a concrete sequence of $\lambda$-values the parameter plane $(\alpha, \gamma)$ is ran through. In particular in each of these case studies the number of phase portraits with $i$ singularities is $N_{i}$, where $\sum_{i=1}^{7} N_{i}$ and $N=L^{+}$(see Theorem 5). Furthermore the study developed in this paper of which the theoretic and numerical results also are confirmed by the computer software package $P 4$ (see [10]), reinforces the idea that the other 4 phase portraits do not occur whatever the value of the parameter. Notice that as a consequence, including the numerical study mentioned above for the 
case of four singularities, the 6-parameter family (1) can have at most 64 topologically different phase portraits. Furthermore based on the case studies it seems that the lower bound in Corollary 3 is the exact total number of topologically different phase portraits.

\section{Parameter Space}

As mentioned before the differential equations left to classify define a subfamily of the reversible vector fields in (1). This subfamily is found to have the following normal form:

$$
X_{(g, \xi, e)}^{R} \leftrightarrow\left\{\begin{array}{l}
\dot{x}=-y+(\xi-2 g) x y-y\left(x^{2}+y^{2}\right), \\
\dot{y}=x+e x^{2}+g y^{2}+x\left(x^{2}+y^{2}\right),
\end{array}\right.
$$

where the parameter coordinates $\xi, g, e \in \mathbb{R}$ correspond to the notation used in [8] and to the respective parameter coordinates $a, b, c$ used in [9]; notice however that these coordinates $a, b, c$ do not correspond to the ones that appear in (1). In [8] the vector field (5) is denoted by $X_{(g, \xi, e)}^{R}$ while in [9] by $X_{(a, b, c)}$. Here we recall the notation of [8] since we want to detail on a result about the Hamiltonian reversible vector fields that have been stated there. However throughout this paper we work with another representation that simplifies both the statement of the results as well as the calculations.

The global phase portraits of (5) having only collinear singularities or infinitely many are classified in [9], as is the case for parameter values satisfying $(2 g-\xi-e) g \leq 0$ or $2 g-\xi=0$. For $(2 g-\xi-e) g>0$ and $2 g-\xi \neq 0$ the vector field $X_{(g, \xi, e)}^{R}$ has at most seven singularities that are generally not collinear, but spread over at most three lines passing through the origin. For a systematic study of the corresponding phase portraits, it is convenient to introduce the new parameter coordinates $(\alpha, \gamma, \lambda)$ and to study $Y_{(\alpha, \gamma, \lambda)}$ which is in one-to-one correspondence with $X_{(g, \xi, e)}^{R}$ in case $(2 g-\xi-e) g>0$. In fact using the transformations

$$
\begin{aligned}
& \mathcal{T}(g, \xi, e)=\left(\sqrt{\frac{-g}{\xi-2 g+e}},-\xi+2 g,-\xi+2 g-e\right) \text { for }(\xi-2 g+e) g<0, \text { and } \\
& \mathcal{Q}(\alpha, \gamma, \lambda)=\left(\alpha^{2} \lambda, 2 \alpha^{2} \lambda-\gamma, \gamma-\lambda\right) \text { for } \alpha>0, \lambda \neq 0,
\end{aligned}
$$

one has the following relations between the different representations for (2),

$$
Y_{(\alpha, \gamma, \lambda)}=X_{\mathcal{Q}(\alpha, \gamma, \lambda)}^{R} \text { and } X_{(g, \xi, e)}^{R}=Y_{\mathcal{T}(g, \xi, e)} \text { and } X_{(a, b, c)}=Y_{\mathcal{T}(b, a, c)},
$$

that are of interest in Sections 3.1 and 5 where results are used from [9] and [8] respectively.

The geometric meaning of the parameter $(\alpha, \gamma, \lambda)$ becomes clear in the study of the singularities of $Y_{(\alpha, \gamma, \lambda)}$ in polar coordinates, defined by $x=r \cos \theta, y=r \sin \theta$. Then the vector field $Y_{(\alpha, \gamma, \lambda)}$ is transformed into the form

$$
\begin{aligned}
& r^{\prime}=r^{2} A(\theta), \\
& \theta^{\prime}=1+B(\theta) r+r^{2},
\end{aligned}
$$

for some cubic homogeneous trigonometric polynomials $A$ and $B$ with coefficients depending on the parameter $(\alpha, \gamma, \lambda)$; in particular, $A$ and $B$ read as

$$
\begin{aligned}
& A(\theta)=\lambda \sin \theta(\alpha \sin \theta-\cos \theta)(\alpha \sin \theta+\cos \theta), \\
& B(\theta)=\cos \theta\left[\left(\alpha^{2} \lambda+\gamma\right) \sin ^{2} \theta+(\gamma-\lambda) \cos ^{2} \theta\right] .
\end{aligned}
$$

From (8) and (9) the geometric meaning of the parameter $\alpha$ is clear. It represents the symmetric rays along which singularities of $Y_{(\alpha, \gamma, \lambda)}$ can be carried. 
By (7) it follows that $Y_{(0, \gamma, \lambda)}=X_{(0,-\gamma, \gamma-\lambda)}^{R}, Y_{(-\alpha, \gamma, \lambda)}=Y_{(\alpha, \gamma, \lambda)}, Y_{(\alpha, 0, \lambda)}=X_{\left(\alpha^{2} \lambda, 2 \alpha^{2} \lambda,-\lambda\right)}^{R}$ and $Y_{(\alpha, \gamma, 0)}=X_{(0,-\gamma, \gamma)}^{R}$. Hence to study the phase portraits of $Y_{(\alpha, \gamma, \lambda)}$ having finitely many noncollinear singularities, it suffices to study the vector fields $Y_{(\alpha, \gamma, \lambda)}$ for

$$
\alpha>0, \gamma \neq 0 \text { and } \lambda \neq 0 \text {. }
$$

Lemma 6. Let $Y_{(\alpha, \gamma, \lambda)}$ be defined in (2). Then we have

(1) $Y_{(\alpha, \gamma, \lambda)}=Y_{(-\alpha, \gamma, \lambda)}$, for all $\alpha, \gamma, \lambda \in \mathbb{R}$.

(2) $Y_{(\alpha, \gamma, \lambda)}$ is invariant with respect to the transformations

$$
\begin{aligned}
& (x, y, t, \alpha, \gamma, \lambda) \mapsto(-x,-y, t, \alpha,-\gamma,-\lambda), \\
& (x, y, t, \alpha, \gamma, \lambda) \mapsto(x,-y,-t, \alpha, \gamma, \lambda) .
\end{aligned}
$$

The invariance property (12) is the one of reversibility: the phase portrait of $Y_{(\alpha, \gamma, \lambda)}$ is symmetric with respect to the horizontal axis when reversing time.

(3) $Y_{(\alpha, \gamma, \lambda)}$ is Hamiltonian if and only if $\gamma=2 \alpha^{2} \lambda$.

By (10) and (11) we can assume throughout this paper that $\alpha, \gamma>0$, it is to say $(\alpha, \gamma, \lambda) \in \mathcal{P}$. In fact, since the phase portraits of $Y_{(\alpha, \gamma, \lambda)}$ with $\gamma<0$ are linearly equivalent to $Y_{(\alpha,-\gamma,-\lambda)}$, the results in Theorems 4 and 5 are evenly valid when replacing $\mathcal{P}_{-}$and $\mathcal{P}_{+}$respectively by $\{(\alpha, \gamma, \lambda): \gamma \lambda<0\}$ and $\{(\alpha, \gamma, \lambda): \gamma \lambda>0\}$.

\section{Singularities}

In this section we study the possible location and type of singularities of $Y_{\zeta}$. From (8) and (9) candidate singularities $(x, y)=(r \cos \theta, r \sin \theta)$ of $Y_{\zeta}$ satisfy $\sin \theta=0$ or $\cot \theta= \pm \alpha$. Furthermore $r$ is solution of the quadratic equation $1+B(\theta) r+r^{2}=0$. Since $\alpha>0$ we can take the angle $\theta_{1}$ for which

$$
0<\theta_{1}<\pi / 2 \text { and } \cot \theta_{1}=\alpha .
$$

In particular $\cos \left(\pi-\theta_{1}\right)=-\alpha / \sqrt{\alpha^{2}+1}$ and $\sin \left(\pi-\theta_{1}\right)=1 / \sqrt{\alpha^{2}+1}$; therefore

$$
B(0)=\gamma-\lambda, A^{\prime}\left(\pi-\theta_{1}\right)=\frac{-2 \alpha \lambda}{\sqrt{\alpha^{2}+1}} \text { and } B\left(\pi-\theta_{1}\right)=\frac{-\alpha \gamma}{\sqrt{\alpha^{2}+1}} \leq 0 .
$$

Clearly, for $\zeta$ fixed, it follows from the reversibility property and (14) that the origin is a center of $Y_{\zeta}$ and all the other singularities appear along the rays $\mathcal{R}_{0}$ and $\mathcal{R}_{ \pm}$, that are defined as

$$
\begin{aligned}
\mathcal{R}_{0} & =\{(x, 0):(\lambda-\gamma) x>0\}, \\
\mathcal{R}_{ \pm} & =\left\{(r, \theta): \theta=\pi \mp \theta_{1}, r>0\right\}=\{(x, y): y=\mp x / \alpha, x<0\} .
\end{aligned}
$$

Notice that none of the rays contain the origin and by the choice $\gamma>0$ the rays $\mathcal{R}_{ \pm}$are contained in the negative $x$-plane. From [8] we know that there are no singularities at infinity. These observations are summarized in the following lemma.

Lemma 7. Let $\zeta \in \mathcal{P}$ and $\mathcal{R}_{0}, \mathcal{R}_{ \pm}$be defined in (15). Then the following properties hold.

(1) The vector field $Y_{\zeta}$ has at most seven singularities of which one is the center at the origin and all singularities are finite.

(2) There are at most three rays that carry singularities of $Y_{\zeta}$, namely the rays $\mathcal{R}_{0}$ and $\mathcal{R}_{ \pm}$. Each of them carry at most two singularities; in particular the singularities on $\mathcal{R}_{+}$and $\mathcal{R}_{-}$are symmetric with respect to the horizontal axis. 
(3) Let the configurations of singularities on the rays $\mathcal{R}_{0}$ and $\mathcal{R}_{ \pm}$be denoted by $(i, j), 0 \leq i, j \leq 2$ if $Y_{\zeta}$ has $1+i+2 j$ singularities in the global phase portrait, of which $i$ lie along $\mathcal{R}_{0}$ and $j$ along $\mathcal{R}_{+}$(as many as along $\mathcal{R}_{-}$). The following configurations are possible:

\begin{tabular}{c|c|c|c|c|c|c|c}
$1+i+2 j$ & 1 & 2 & 3 & 4 & 5 & 6 & 7 \\
\hline$(i, j)$ & $(0,0)$ & $(1,0)$ & $(2,0),(0,1)$ & $(1,1)$ & $(2,1),(0,2)$ & $(1,2)$ & $(2,2)$
\end{tabular}

The precise location and topological type of the singularities along $\mathcal{R}_{0}$ (resp. along $\mathcal{R}_{ \pm}$) are described in Proposition 8 (resp. Propositions 9, 10 and 11).

3.1. Singularities along $\mathcal{R}_{0}$. Recall that $\mathcal{R}_{0}$ is the horizontal ray as defined in (15) and that in [9] singularities along $\mathcal{R}_{0}$ are completely analyzed for $X_{(a, b, c)}$ defined in (7). Then the topological type of the singularities of $Y_{\zeta}$ along $\mathcal{R}_{0}$ depends only on $\gamma-\lambda$ and is stated in the proposition below.

Proposition 8. Let $\zeta \in \mathcal{P}$. Then the local phase portrait of (2) along $\mathcal{R}_{0}$ is sketched in Figures 3 and 4 and is described as follows:

(1) If $\zeta \in \mathcal{E}_{u} \cap \mathcal{F}_{d}$, then there are no singularities along $\mathcal{R}_{0}$.

(2) If $\zeta \in \mathcal{E} \cup \mathcal{F}$, then there is only a cusp singularity at $\mathbf{s}^{0}=(-\operatorname{sgn}(\gamma-\lambda), 0)$. Its direction is determined by the sign of $\lambda$.

(3) If $\zeta \in \mathcal{E}_{d} \cup \mathcal{F}_{u}$, then there are two singularities, given by $\mathbf{s}_{ \pm}^{0}=\left(-\operatorname{sgn}(\gamma-\lambda) r_{ \pm}^{0}, 0\right)$, where $r_{ \pm}^{0}$ is the function of $\gamma-\lambda$ :

$$
r_{ \pm}^{0}=\frac{|\gamma-\lambda| \pm \sqrt{(\gamma-\lambda)^{2}-4}}{2} .
$$

Furthermore, $\mathbf{s}_{\sigma_{1}}^{0}$ is a saddle and $\mathbf{s}_{-\sigma_{1}}^{0}$ is a center where $\sigma_{1}=\operatorname{sgn}(\lambda) \operatorname{sgn}(\gamma-\lambda)$.

We conclude that 5 different possibilities for the local phase portraits along the horizontal axis are distinguished; restricting to $\mathcal{P}_{-}$only 3 of them are encountered since $\mathcal{P} \subset\{\gamma>0\}$. This is presented in Figures 3 and 4 in which furthermore the relative position of the separatrices at the singularities of saddle or cusp type with respect to $\mathcal{R}_{0}$ is shown. To distinguish different separatrices at a singularity s along $\mathcal{R}_{0}$ of saddle or cusp type, we denote the stable and unstable manifolds at $\mathbf{s}$ by $\mathcal{W}(\mathbf{s})$ and $\mathcal{U}(\mathbf{s})$ respectively. Moreover we introduce the subsets $\mathcal{W}_{I}(\mathbf{s}) \subset \mathcal{W}(\mathbf{s}) \backslash\{\mathbf{s}\}$ and $\mathcal{U}_{I}(\mathbf{s}) \subset \mathcal{U}(\mathbf{s}) \backslash\{\mathbf{s}\}$ for the respective subsets having their germ at $\mathbf{s}$ included in $\{(x, y): y<0\}$; analogously we introduce $\mathcal{W}_{E}(\mathbf{s}) \subset \mathcal{W}(\mathbf{s}) \backslash\{\mathbf{s}\}$ and $\mathcal{U}_{E}(\mathbf{s}) \subset \mathcal{U}(\mathbf{s}) \backslash\{\mathbf{s}\}$ for the respective subsets having their germ at $\mathbf{s}$ included in $\{(x, y): y>0\}$. This notation is used in Figures 12, 13, 14, 18, 19 and 29, where the different possible relative positions for the stable and unstable manifolds are sketched.

3.2. Singularities on $\mathcal{R}_{ \pm}$. Recall that $\mathcal{R}_{ \pm}$are the symmetric rays defined in (15). Due to the symmetry of $Y_{\zeta}$ we only need to study the singularities on $\mathcal{R}_{+}$. The singularities on $\mathcal{R}_{-}$and their type can be obtained directly using the reversibility property. Moreover, the singularities occur in pairs: singularities on $\mathcal{R}_{+}$are reflected on $\mathcal{R}_{-}$with respect to the $x$-axis, and viceversa. The number, location and topological type of the singularities of $Y_{\zeta}$ along $\mathcal{R}_{+}$are described in Propositions 9, 10 and 11, where the local bifurcation diagram is found to be determined by the surfaces $\mathcal{G}, \mathcal{G}_{l}, \mathcal{G}_{r}, \mathcal{H}, \mathcal{H}_{l}$ and $\mathcal{H}_{r}$ as introduced in (4). The following proposition follows from straightforward calculations. 


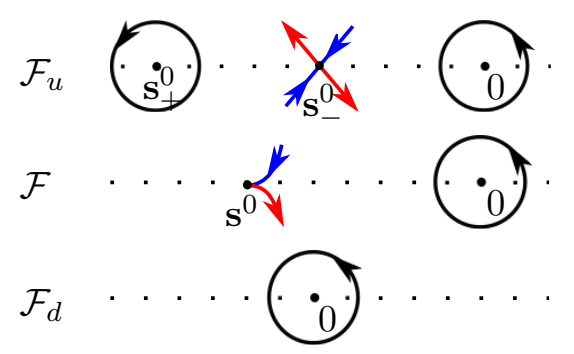

FiguRe 3. $\mathcal{F}_{u}, \mathcal{F}, \mathcal{F}_{d}$ form a partition of $\mathcal{P}_{-}$defined by different configurations of singularities along $\mathcal{R}_{0}$, see Proposition 8 .

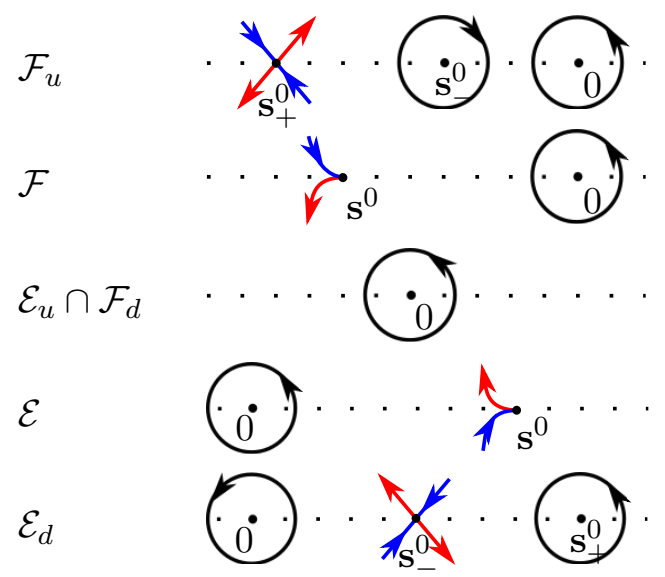

FiguRE 4. $\mathcal{F}_{u}, \mathcal{F}, \mathcal{F}_{d} \cap \mathcal{E}_{u}, \mathcal{E}, \mathcal{E}_{d}$ form a partition of $\mathcal{P}_{+}$defined by the different configurations of singularities along $\mathcal{R}_{0}$, see Proposition 8 .

Proposition 9. Let $\zeta \in \mathcal{P}$.

(1) If $\zeta \in \mathcal{G}_{l}$, then the vector field $Y_{\zeta}$ has no singularities on $\mathcal{R}_{+}$.

(2) If $\zeta \in \mathcal{G}$, then the vector field $Y_{\zeta}$ has one singularity on $\mathcal{R}_{+}$, that we denote by $\mathbf{s}^{1}=\left(x^{1}, y^{1}\right)$. In particular,

$$
\begin{gathered}
\mathbf{s}^{1}=1 / \sqrt{\alpha^{2}+1} \cdot(-\alpha, 1), r^{1}=\left\|\mathbf{s}^{1}\right\|=1, \\
\alpha=-x^{1} / y^{1} \text { and } \gamma=-\left(\left(x^{1}\right)^{2}+\left(y^{1}\right)^{2}+1\right) / x^{1} .
\end{gathered}
$$

(3) If $\zeta \in \mathcal{G}_{r}$, then the vector field $Y_{\zeta}$ has two singularities on $\mathcal{R}_{+}$, that we denote by $\mathbf{s}_{ \pm}^{1}$. In particular,

$$
\begin{gathered}
\mathbf{s}_{ \pm}^{1}=y_{ \pm}^{1} \cdot(-\alpha, 1), \text { where } y_{ \pm}^{1}=\frac{\alpha \gamma \pm \sqrt{\alpha^{2} \gamma^{2}-4\left(\alpha^{2}+1\right)}}{2\left(\alpha^{2}+1\right)}=\frac{r_{ \pm}^{1}}{\sqrt{\alpha^{2}+1}} \\
r_{ \pm}^{1}=\left\|\mathbf{s}_{ \pm}^{1}\right\|=\frac{\alpha \gamma \pm \sqrt{\alpha^{2} \gamma^{2}-4\left(\alpha^{2}+1\right)}}{2 \sqrt{\alpha^{2}+1}}, 0<r_{-}^{1}<1<r_{+}^{1}, \\
\alpha=-x_{+}^{1} / y_{+}^{1} \text { and } \gamma=-\left(\left(x_{+}^{1}\right)^{2}+\left(y_{+}^{1}\right)^{2}+1\right) / x_{+}^{1} .
\end{gathered}
$$

Analogously as in Proposition 9 the singularities on $\mathcal{R}_{-}$are denoted by $\mathbf{s}_{ \pm}^{2}$ or $\mathbf{s}^{2}$, and by symmetry are determined by

$$
\mathbf{s}^{2}=1 / \sqrt{\alpha^{2}+1} \cdot(-\alpha,-1) \text { and } \mathbf{s}_{ \pm}^{2}=y_{ \pm}^{1} \cdot(-\alpha,-1) .
$$


Proposition 10. Let $\zeta \in \mathcal{G}_{r} \cap \mathcal{P}$ and let $\mathbf{s}_{ \pm}^{1}$ and $y_{ \pm}^{1}$ be as defined in (18). Then the singularities $\mathbf{S}_{ \pm}^{1}$ are hyperbolic and are classified as follows:

\begin{tabular}{l|l|l} 
& $\mathbf{s}_{-}^{1}$ & $\mathbf{s}_{+}^{1}$ \\
\hline in $\mathcal{G}_{r} \cap \mathcal{P}_{+}$ & saddle & stable node or focus in $\mathcal{H}_{l}$, \\
& & $\begin{array}{l}\text { Hamiltonian center in } \mathcal{H} \text { and } \\
\text { unstable node or focus in } \mathcal{H}_{r}\end{array}$ \\
\hline in $\mathcal{G}_{r} \cap \mathcal{P}_{-}$ & stable node or focus & saddle
\end{tabular}

The trace $\operatorname{tr}$ and determinant $\mathrm{D}$ of the linearization of (2) at $\mathbf{s}_{ \pm}^{1}$ are given by

$$
\operatorname{tr}\left(\mathbf{s}_{ \pm}^{1}\right)=\left(2 \alpha^{2} \lambda-\gamma\right) y_{ \pm}^{1} \text { and } \mathrm{D}\left(\mathbf{s}_{ \pm}^{1}\right)= \pm 2 \alpha \lambda\left(y_{ \pm}^{1}\right)^{2} \sqrt{\alpha^{2} \gamma^{2}-4\left(\alpha^{2}+1\right)} .
$$

In particular $\operatorname{sgn}\left(\operatorname{tr}\left(\mathbf{s}_{ \pm}^{1}\right)\right)=\operatorname{sgn}\left(2 \alpha^{2} \lambda-\gamma\right)$ and $\operatorname{sgn}\left(\mathrm{D}\left(\mathbf{s}_{ \pm}^{1}\right)\right)= \pm \operatorname{sgn}(\lambda)$. For $\lambda \neq 0$ the tangent vectors to the separatrices at the saddle point $\mathbf{s}_{\sigma_{2}}^{1}$ where $\sigma_{2}=\operatorname{sgn}(-\lambda)$ are given by

$$
\mathbf{v}_{ \pm}^{1, \sigma_{2}}=\left(2\left(y_{\sigma_{2}}^{1}\right)^{2},-\mu_{ \pm}^{1, \sigma_{2}}+\left(2 \alpha y_{\sigma_{2}}^{1}-\gamma\right) y_{\sigma_{2}}^{1}\right)
$$

and the direction of the flow on the corresponding separatrices is determined by

$$
\mu_{ \pm}^{1, \sigma_{2}}=\left(-\gamma+\frac{1}{2}\left(\gamma+2 \alpha^{2} \lambda\right) \pm \frac{1}{2} \sqrt{\left(\gamma+2 \alpha^{2} \lambda\right)^{2}-16 y_{\sigma_{2}}^{1} \alpha\left(\alpha^{2}+1\right) \lambda}\right) y_{\sigma_{2}}^{1} .
$$

Proof. The statement about the singularities $\mathbf{s}_{ \pm}^{1}$ in case $\gamma>2 \sqrt{\alpha^{2}+1} / \alpha$ is based on the classification of the trace and determinant at their linearizations; the formulas in (21) follow from direct calculation. To determine the asymptotics of the separatrices at the saddle point $\mathbf{s}_{\sigma_{2}}^{1}$, one can calculate the eigenvalues $\mu_{ \pm}^{1, \sigma_{2}}$ and corresponding eigenvectors $\mathbf{v}_{ \pm}^{1, \sigma_{2}}$ of the linearization $J$ at the singularity $\mathbf{s}_{\sigma_{2}}^{1}$ :

$$
J\left(\mathbf{s}_{\sigma_{2}}^{1}\right)=\left[\begin{array}{cc}
y_{\sigma_{2}}^{1}\left(-\gamma+2 \alpha y_{\sigma_{2}}^{1}\right) & -2\left(y_{\sigma_{2}}^{1}\right)^{2} \\
\alpha y_{\sigma_{2}}^{1}\left(2 \alpha y_{\sigma_{2}}^{1}+2 \lambda-\gamma\right) & 2 \alpha y_{\sigma_{2}}^{1}\left(-y_{\sigma_{2}}^{1}+\alpha \lambda\right)
\end{array}\right]
$$

where we use the fact that $x_{\sigma_{2}}^{1}=-\alpha y_{\sigma_{2}}^{1}$ and $1+\gamma x_{\sigma_{2}}^{1}+\left(x_{\sigma_{2}}^{1}\right)^{2}+\left(y_{\sigma_{2}}^{1}\right)^{2}=0$.

Furthermore to distinguish between different separatrices at the saddle singularity or saddle-node along $\mathcal{R}_{ \pm}$, we introduce the open sets $I$ and $E$ such that

$$
\begin{aligned}
& I \cap E=\emptyset, \mathbb{R}^{2}=I \cup E \cup\left(\mathcal{R}_{+} \cup \mathcal{R}_{-} \cup\{(0,0)\}\right), \\
& I \supset\{(x, 0): x<0\} \text { and } E \supset\{(x, 0): x>0\} .
\end{aligned}
$$

In Propositions 10 and 11 the relative position and tangency of the separatrices of the singularities along $\mathcal{R}_{ \pm}$is detailed precisely like in Figures 5 and 6 ; in particular in which region $I$ or $E$ they start. Moreover these regions are used to describe possible connections between singularities later in this paper. If the stable and unstable manifolds at a saddle or saddle-node $\mathbf{s}$ along $\mathcal{R}_{ \pm}$are denoted by $\mathcal{W}(\mathbf{s})$ and $\mathcal{U}(\mathbf{s})$ respectively, then we denote by $\mathcal{W}_{I}(\mathbf{s}) \subset \mathcal{W}(\mathbf{s}) \backslash\{\mathbf{s}\}$ and $\mathcal{U}_{I}(\mathbf{s}) \subset \mathcal{U}(\mathbf{s}) \backslash\{\mathbf{s}\}$ the respective subsets having their germ at $\mathbf{s}$ in $I$. Analogously we denote by $\mathcal{W}_{E}(\mathbf{s}) \subset \mathcal{W}(\mathbf{s}) \backslash\{\mathbf{s}\}$ and $\mathcal{U}_{E}(\mathbf{s}) \subset \mathcal{U}(\mathbf{s}) \backslash\{\mathbf{s}\}$ for the respective subsets having their germ at $\mathbf{s}$ in $E$. This notation is used in Figures 12, $13,14,18,19$ and 29. 


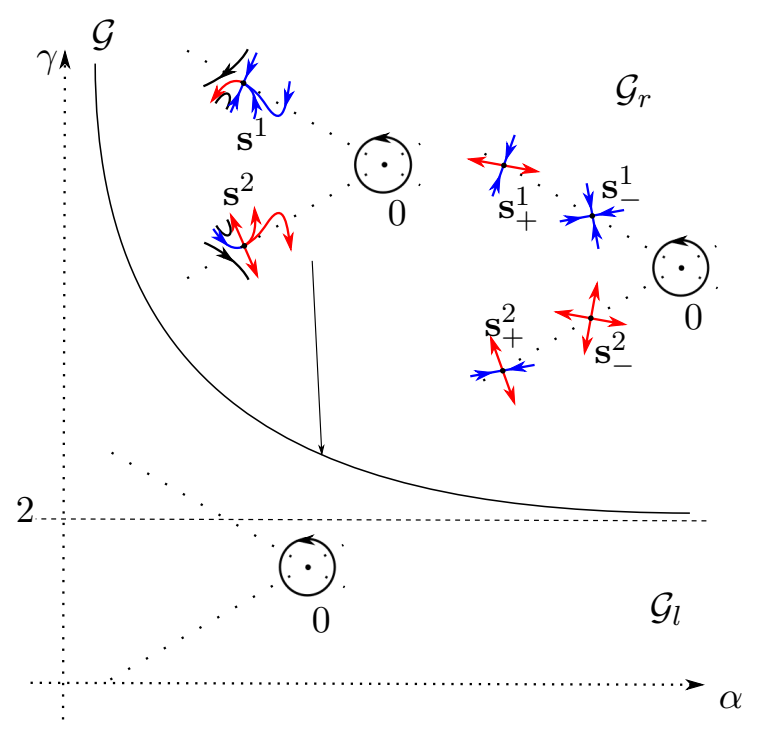

FiguRE 5. $\mathcal{G}_{l}, \mathcal{G}, \mathcal{G}_{r}$ form a partition of $\mathcal{P}_{-}$defined by different configurations of singularities along $\mathcal{R}_{ \pm}$, see Propositions 9, 10 and 11.

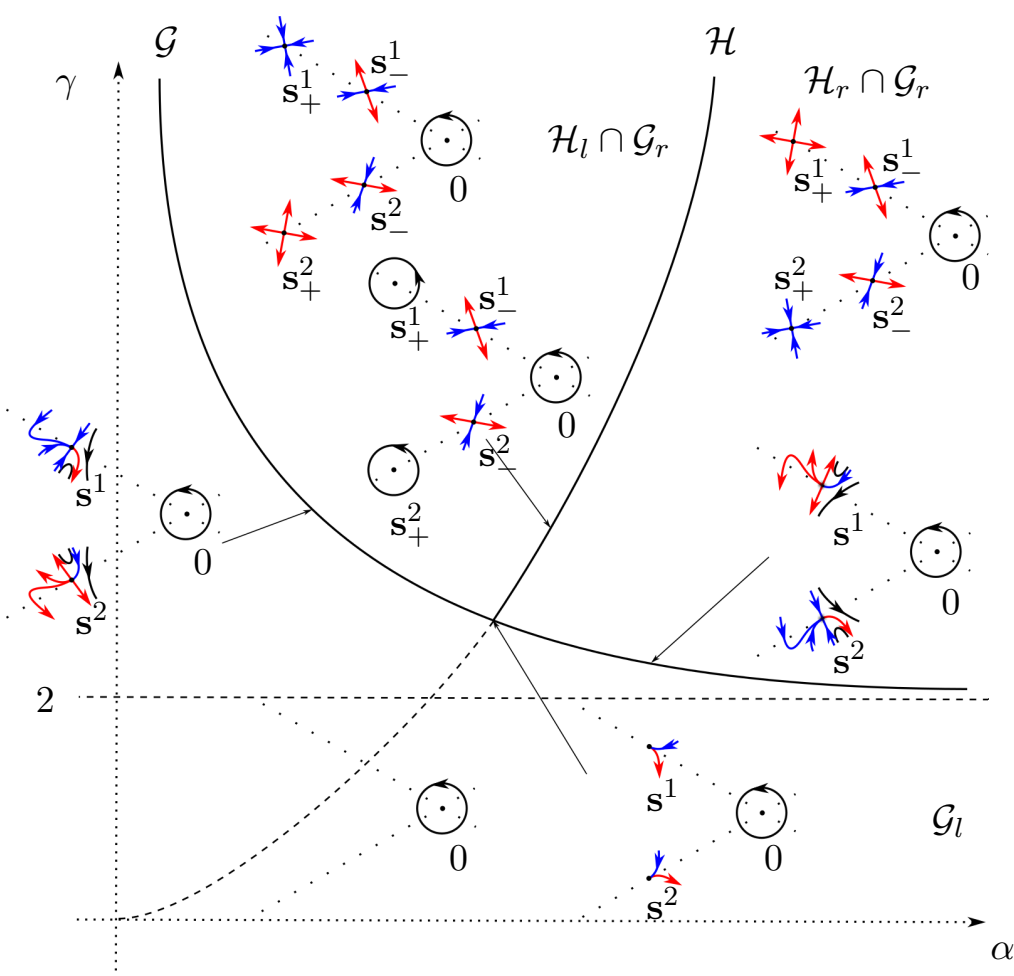

FiguRE 6. $\mathcal{G}_{l}, \mathcal{G} \cap \mathcal{H}_{r}, \mathcal{G} \cap \mathcal{H}, \mathcal{G} \cap \mathcal{H}_{l}, \mathcal{G}_{r} \cap \mathcal{H}_{r}, \mathcal{G}_{r} \cap \mathcal{H}, \mathcal{G}_{r} \cap \mathcal{H}_{l}$ form a partition of $\mathcal{P}_{+}$defined by different configurations of singularities along $\mathcal{R}_{ \pm}$, see Propositions 9, 10 and 11. 
Proposition 11. Let $\zeta \in \mathcal{G} \cap \mathcal{P}$ and let $r, \mathbf{s}^{1}=\left(x^{1}, y^{1}\right), I$ and $E$ be defined in (16) and (22). Then $\gamma=2 \sqrt{\alpha^{2}+1} / \alpha$ and the trace $\operatorname{tr}$ and determinant $\mathrm{D}$ of the linearization of (2) at the singularity $\mathbf{s}^{1}$ are given by

$$
\operatorname{tr}\left(\mathbf{s}^{1}\right)=2 \alpha^{2}\left(\lambda-l_{\alpha}\right) / \sqrt{\alpha^{2}+1}=2\left(\lambda\left(x^{1}\right)^{3}+\left(y^{1}\right)^{2}\right) /\left(x^{1} y^{1}\right) \text { and } \mathrm{D}\left(\mathbf{s}^{1}\right)=0,
$$

where

Then,

$$
l_{\alpha}=\frac{\sqrt{\alpha^{2}+1}}{\alpha^{3}}>0
$$

(1) $\mathcal{H} \cap \mathcal{G} \cap \mathcal{P}=\left\{\left(\alpha, 2 \alpha^{2} l_{\alpha}, l_{\alpha}\right): \alpha>0\right\}=\left\{\left(\alpha_{0}(\lambda), 2\left(\alpha_{0}(\lambda)\right)^{2} \lambda, \lambda\right): \lambda>0\right\}$, where the analytic curve $\alpha_{0}:(0, \infty) \rightarrow(0, \infty)$ is defined by the positive solution $\alpha_{0}(\lambda)$ of $\lambda^{2} \alpha^{6}-\alpha^{2}-1=0$. In particular, if $\zeta \in \mathcal{H} \cap \mathcal{G} \cap \mathcal{P}$, then $\mathbf{s}^{1}$ is a nilpotent cusp whose separatrices can asymptotically be parameterized by

$$
\begin{aligned}
& u \mapsto\left(-\frac{\alpha}{\sqrt{\alpha^{2}+1}}+u^{2}, \frac{1}{\sqrt{\alpha^{2}+1}}-\frac{1}{\alpha} u^{2}+\varphi_{ \pm}(\alpha) u^{3}+O\left(u^{4}\right)\right), u \rightarrow 0, \\
& \text { where } \varphi_{ \pm}(\alpha)= \pm\left(\alpha^{2}+1\right)^{5 / 4} / \sqrt{3 \alpha^{3}}= \pm 1 /\left(\sqrt{3\left(x^{1}\right)^{3}} y^{1}\right) \text {. }
\end{aligned}
$$

(2) If $\zeta \in(\mathcal{G} \cap \mathcal{P}) \backslash \mathcal{H}$, then $\mathbf{s}^{1}$ is a semi-hyperbolic saddle-node, whose separatrices can asymptotically be parameterized by

$$
\begin{aligned}
& u \mapsto\left(x^{1}+u^{2}, y^{1}+\phi_{1}(\alpha) u^{2}+\phi_{2}(\alpha, \lambda) u^{4}+O\left(u^{5}\right)\right), \text { for } u \rightarrow 0 \text { and } \\
& u \mapsto\left(x^{1}+u^{2}, y^{1}+\phi_{3}(\alpha, \lambda) u^{2}+O\left(u^{3}\right)\right), \text { for } u \rightarrow 0,
\end{aligned}
$$

where $\phi_{1}(\alpha)=-1 / \alpha=y^{1} / x^{1}$,

$$
\begin{aligned}
& \phi_{2}(\alpha, \lambda)=\alpha l_{\alpha}^{2} /\left[2\left(\lambda-l_{\alpha}\right)\right]=-\left(y^{1}\right)^{3} /\left[2\left(x^{1}\right)^{2}\left(\lambda\left(x^{1}\right)^{3}+\left(y^{1}\right)^{2}\right)\right] \text { and } \\
& \phi_{3}(\alpha, \lambda)=\alpha\left(1-\alpha \lambda \sqrt{\alpha^{2}+1}\right)=-x^{1}\left(\lambda x^{1}+\left(y^{1}\right)^{2}\right) /\left(y^{1}\right)^{3} .
\end{aligned}
$$

The relative position and tangency of the separatrices (24), (25) and (26) with respect to $\mathcal{R}_{+}, I$ and $E$, and the direction of the flow on these separatrices is as in Figure 5 for $\lambda<0$ and Figure 6 for $\lambda>0$.

Proof. Since the function $(0, \infty) \rightarrow(0, \infty): \alpha \mapsto l_{\alpha}$ defined by (23) is strictly decreasing with $\alpha$, it has an analytic inverse, that we call by $\alpha_{0}$. As a consequence, along the curve $\gamma=2 \sqrt{\alpha^{2}+1} / \alpha$, there is a unique $\alpha_{0}$ such that $l_{\alpha_{0}}=\sqrt{\alpha_{0}^{2}+1} / \alpha_{0}^{3}$. For all $\zeta \in \mathcal{G} \cap \mathcal{H}_{l}=\mathcal{G} \cap\left\{\alpha<\alpha_{0}\right\}$ it follows that $\lambda>l_{\alpha_{0}}$ and for all $\zeta \in \mathcal{G} \cap \mathcal{H}_{r}=\mathcal{G} \cap\left\{\alpha>\alpha_{0}\right\}$ it follows that $\lambda<l_{\alpha_{0}}$.

To analyze the topological type of the singularity $\mathbf{s}^{1}$ in $\mathcal{P} \cap \mathcal{G}$, we use [9] and Topological Normal Form Theorems for semi-hyperbolic and nilpotent singularities (see e.g. $[1,10])$. Let $\zeta \in \mathcal{P} \cap \mathcal{G}$ be fixed. A direct calculation shows that

$$
J\left(\mathbf{s}^{1}\right)=\frac{1}{\alpha^{2}+1}\left[\begin{array}{cc}
-2 / \alpha & -2 \\
2\left(\lambda \alpha \sqrt{\alpha^{2}+1}-1\right) & 2 \alpha\left(\lambda \alpha \sqrt{\alpha^{2}+1}-1\right)
\end{array}\right] ;
$$

therefore

$$
\mu=\operatorname{tr}\left(\mathbf{s}^{1}\right)=\frac{2 \lambda \alpha^{2}}{\sqrt{\alpha^{2}+1}}-\frac{2}{\alpha} \text { and } \mathrm{D}\left(\mathbf{s}^{1}\right)=0 .
$$

If $\lambda \neq l_{\alpha}$, then $\mu \neq 0$ and hence $\mathbf{s}^{1}$ is semi-hyperbolic. Notice that when $\lambda<0$ this is always the case and then $\mu<0$. If $\lambda=l_{\alpha}$, then $\mathbf{s}^{1}$ is nilpotent and needs a separate study. 
Suppose first that $\lambda \neq l_{\alpha}$, then we reduce (2) to the topological normal form for semi-hyperbolic singularities, i.e. $\dot{u}=f(u, v), \dot{v}=\mu v+g(u, v)$, where $f$ and $g$ are $O\left(\|(u, v)\|^{2}\right)$ for $\|(u, v)\| \rightarrow 0$, by the transformation

$$
(u, v)=C^{-1}\left(x+\frac{\alpha}{\sqrt{\alpha^{2}+1}}, y-\frac{1}{\sqrt{\alpha^{2}+1}}\right) \text {, where } C=\left[\begin{array}{cc}
-\alpha & 1 \\
1 & \alpha\left(1-\lambda \alpha \sqrt{\alpha^{2}+1}\right)
\end{array}\right] .
$$

For any curve $\mathcal{C}$ along which $\dot{v}$ vanishes identically, its asymptotics for $u \rightarrow 0$ is given by $v=-\frac{1}{2} \alpha\left(\alpha^{2}+1\right)^{3 / 2}\left(-\alpha^{3} \lambda+\alpha^{2}+1\right)^{-2} u^{2}+O\left(u^{3}\right)$. As such the asymptotics of $f$ restricted to $\mathcal{C}$ is given by $\rho u^{2}+O\left(u^{3}\right), u \rightarrow 0$, with

$$
\rho=\frac{\alpha^{3} \lambda\left(\alpha^{2}+1\right)}{\alpha \sqrt{\alpha^{2}+1}\left(\alpha^{3} \lambda-\sqrt{\alpha^{2}+1}\right)} .
$$

For $\lambda<0$ it follows that $\rho>0$ and $\mu<0$. For $\lambda>l_{\alpha}$ one has $\rho, \mu>0$ and for $0<\lambda<l_{\alpha}$ one has $\rho, \mu<0$. From the Topological Normal Form Theorem for semihyperbolic singularities it then follows that $\mathbf{s}^{1}=\left(x^{1}, y^{1}\right)$ is a saddle-node with the local phase portraits drawn in Figures 5 and 6 . Furthermore it can easily be checked that the separatrices at $\mathbf{s}^{1}$ in the coordinates $(x, y)$ has the asymptotics given in (25) and (26).

Next suppose that $\lambda=l_{\alpha}$ and hence $\mathbf{s}^{1}=\left(x^{1}, y^{1}\right)$ is a nilpotent singularity. Then we reduce (2) to the standard normal form for these singularities, $\dot{u}=v+f(u, v), \dot{v}=$ $g(u, v)$, where $f(u, v)=g(u, v)=O\left(\|(u, v)\|^{2}\right),\|(u, v)\| \rightarrow 0$, by the transformation

$$
(x, y) \mapsto C^{-1}\left(x+\frac{\alpha}{\sqrt{\alpha^{2}+1}}, y-\frac{1}{\sqrt{\alpha^{2}+1}}\right) \text {, where } C=\left[\begin{array}{cc}
-\alpha & \frac{1}{2} \alpha^{2}\left(\alpha^{2}+1\right) \\
1 & 0
\end{array}\right] .
$$

For any curve $\mathcal{C}$ along which $\dot{u}$ vanishes identically, its asymptotics for $u \rightarrow 0$ is given by $v=\alpha \sqrt{\alpha^{2}+1} u^{2}+O\left(u^{3}\right)$. As such the asymptotics of $g$ restricted to $\mathcal{C}$ is given by $\rho u^{2}+O\left(u^{3}\right), u \rightarrow 0$, with

$$
\rho=\frac{-2 \sqrt{\alpha^{2}+1}}{\alpha^{2}}<0 \quad \text { and } \quad \frac{\partial f(u, v)}{\partial u}+\left.\frac{\partial g(u, v)}{\partial v}\right|_{(u, v) \in \mathcal{C}}=0 .
$$

Therefore from the Topological Normal Form Theorem for nilpotent singularities it follows that $\mathbf{s}^{1}$ is a cusp in case $\lambda=l_{\alpha}$ (see Figure 6 ). It can easily be checked that the separatrices at $\mathbf{s}^{1}$ in the coordinates $(x, y)$ have the asymptotics given in (24).

To verify the relative position of the separatrices of the saddle-node and its dynamical behavior for $\lambda \neq l_{\alpha}$, we notice that (26) can locally be written as

$$
y=y^{1}+\phi_{3}\left(x-x^{1}\right)+O\left(\left(x-x^{1}\right)^{2}\right), x \rightarrow x^{1},
$$

and hence the behavior on it is asymptotically given by

$$
\dot{y}=\mu\left(y-y^{1}\right)+O\left(\left(y-y^{1}\right)^{2}\right), y \rightarrow y^{1} .
$$

For $\lambda<l_{\alpha}$ (resp. $\lambda>l_{\alpha}$ ) the hyperbolic behavior is attracting (resp. repelling). Furthermore it follows that the non-hyperbolic behavior of the saddle-node happens along the separatrix with parametrization (25). This separatrix is tangent to $\mathcal{R}_{+}$at $\mathbf{s}^{1}$ and locally near $\mathbf{s}^{1}$ concave up (resp. concave down) for $\lambda>l_{\alpha}\left(\right.$ resp. $\left.\lambda<l_{\alpha}\right)$.

To determine the sense of the flow on the separatrices determined by (25) and (26) we consider the sign of $\dot{r}$ for $\theta$ near $\pi-\theta_{1}$ :

$$
\operatorname{sgn}(\dot{r})=\operatorname{sgn}(\lambda) \operatorname{sgn}\left(\theta_{1}-\theta\right) \text { for } \theta \rightarrow \pi-\theta_{1},
$$


since $\cot \left(\pi-\theta_{1}\right)=-\alpha$. Taking this into account we find the saddle-nodes as drawn in Figures 5 and 6 . Similarly the relative position of the separatrices of the cusp and its dynamic behavior is found as drawn in Figure 6.

3.3. Local bifurcation diagram. In this section we combine the results from Sections 3.1 and 3.2 to obtain the local bifurcation diagram in $\mathcal{P}$. It is thus formed by the bifurcation surfaces $\mathcal{E}, \mathcal{F}, \mathcal{G}$ and $\mathcal{H}$ restricted to $\mathcal{P}$ as defined in (3) and (4), i.e. surfaces passing through which the number and/or the topological type of singularities change. All characteristic slices of the local bifurcation diagram for fixed $\lambda<0$ (resp. $\lambda>0$ ) are drawn in Figure 2 (resp. Figure 7 ); the relative position of the bifurcation curves is justified by Lemma 12 below. Let $\pi_{1}, \pi_{2}$ and $\pi_{3}$ denote the canonical projections

$$
\pi_{1}(\alpha, \gamma, \lambda)=(\gamma, \lambda), \pi_{2}(\alpha, \gamma, \lambda)=(\alpha, \lambda) \text { and } \pi_{3}(\alpha, \gamma, \lambda)=(\alpha, \gamma)
$$

A characteristic slice of the bifurcation diagram $\mathcal{P}_{\lambda}$ is composed by four bifurcation curves $\mathcal{E}_{\lambda}, \mathcal{F}_{\lambda}, \mathcal{G}_{\lambda}$ and $\mathcal{H}_{\lambda}$. Passing through these curves the singularities on the horizontal axis appear or disappear through a cusp or split into a center and a saddle, depending on the relative order or mutual intersections of the bifurcation surfaces that are given in Lemma 12. Furthermore the number and topological type of the singularities along $\mathcal{R}_{ \pm}$are controlled by the bifurcation curves $\pi_{3}\left(\mathcal{H}_{\lambda}\right)$ and $\pi_{3}\left(\mathcal{G}_{\lambda}\right)$. Both can be written as graphs $\gamma=\gamma(\alpha), \alpha>0$. The latter one contains the parameter values $(\alpha, \gamma)$ for which the singularity of $Y_{\zeta}$ along $\mathcal{R}_{ \pm}$generically is a saddle-node and the other curve contains parameter values $(\alpha, \gamma)$ for which $Y_{\zeta}$ is Hamiltonian.

Lemma 12. Let $\mathcal{P}, \mathcal{E}, \mathcal{F}, \mathcal{G}, \mathcal{H}$ be as defined in (3) and (4).

(1) $\mathcal{H}_{\lambda}=\emptyset$ if $\lambda<0$.

(2) $\pi_{3}\left(\mathcal{G}_{\lambda}\right)$ is identical for all $\lambda>0: \pi_{3}\left(\mathcal{G}_{\lambda}\right)=\pi_{3}(\mathcal{G})$; as the graph $\gamma=2 \sqrt{\alpha^{2}+1} / \alpha$, $\alpha>0$, it is strictly decreasing with respect to $\alpha$ and it has a horizontal asymptote $\gamma=2$ for $\alpha \rightarrow \infty$ and a vertical asymptote at $\alpha=0$.

(3) $\mathcal{H}_{\lambda_{0}} \cap \mathcal{G}_{\lambda_{0}}$ is empty for $\lambda_{0}<0$ and a singleton $\left\{\left(\alpha_{0}, \gamma_{0}, \lambda_{0}\right)\right\}$ for $\lambda_{0}>0$. Furthermore, the curves $\alpha_{0}:(0, \infty) \rightarrow(0, \infty)$ and $\gamma_{0}:(0, \infty) \rightarrow(2, \infty)$ are analytic and strictly decreasing resp. increasing such that $\alpha_{0}=\alpha\left(\lambda_{0}\right)$ is the positive solution of $\alpha_{0}^{3} \lambda_{0}^{2}-\alpha_{0}^{2}-1=0$ and $\gamma\left(\lambda_{0}\right)=2 \sqrt{1+\alpha_{0}^{2}\left(\lambda_{0}\right)} / \alpha_{0}\left(\lambda_{0}\right)$ with

$$
\lim _{\lambda_{0} \downarrow 0} \gamma_{0}\left(\lambda_{0}\right) \text { and } \lim _{\lambda_{0} \rightarrow \infty} \gamma_{0}\left(\lambda_{0}\right)=\infty \text {. }
$$

(4) The intersection $\mathcal{E} \cap \mathcal{P}_{\lambda}$ is nonempty if and only if $\lambda \geq 2$. In particular,

(a) $\pi_{3}\left(\mathcal{E}_{\lambda}\right)<\pi_{3}\left(\mathcal{G}_{\lambda}\right)$ if $2 \leq \lambda \leq 4$. Equivalently, for all $\alpha>0$ and $\gamma_{e}, \gamma_{g} \geq 0$ with $\left(\alpha, \gamma_{e}, \lambda\right) \in \mathcal{E}_{\lambda}$ and $\left(\alpha, \gamma_{g}, \lambda\right) \in \mathcal{G}_{\lambda}$, it holds that $\gamma_{e} \leq 2<\gamma_{g}$.

(b) $\mathcal{E}_{\lambda} \cap \mathcal{G}_{\lambda}=\left\{\left(\alpha_{+}(\lambda), \gamma_{-}(\lambda), \lambda\right)\right\}$ for some $\alpha_{+}(\lambda)>0$ and $\gamma_{-}(\lambda) \geq 0$ such that $\gamma_{-}(\lambda)<\gamma_{0}(\lambda)$ if $4<\lambda<6$

(c) $\mathcal{E}_{6} \cap \mathcal{G}_{6}=\{(1 / \sqrt{3}, 4,6)\}$.

(d) $\mathcal{E}_{\lambda} \cap \mathcal{G}_{\lambda}=\left\{\left(\alpha_{-}(\lambda), \gamma_{+}(\lambda), \lambda\right)\right\}$ for some $\alpha_{-}(\lambda)>0, \gamma_{+}(\lambda)>0$ such that $\gamma_{0}(\lambda)<\gamma_{+}(\lambda)$ if $\lambda>6$.

(5) The intersection $\mathcal{F}_{\lambda}$ is nonempty if and only if $\lambda \geq-2$. In particular,

(a) $\pi_{3}\left(\mathcal{F}_{\lambda}\right)<\pi_{3}\left(\mathcal{G}_{\lambda}\right)$, if $-2 \leq \lambda<0$. Or, for all $\alpha>0$ and $\gamma_{f}, \gamma_{g} \geq 0$ with $\left(\alpha, \gamma_{f}, \lambda\right) \in \mathcal{F}_{\lambda}$ and $\left(\alpha, \gamma_{g}, \lambda\right) \in \mathcal{G}_{\lambda}$, it holds that $\gamma_{f}<2<\gamma_{g}$.

(b) $\mathcal{F}_{\lambda} \cap \mathcal{G}_{\lambda}=\left\{\left(\alpha_{1}(\lambda), \gamma_{1}(\lambda), \lambda\right)\right\}$, for some $\alpha_{1}(\lambda)>0, \gamma_{1}(\lambda)>0$ such that $\gamma_{0}(\lambda)<\gamma_{1}(\lambda)$ if $\lambda>0$. 
It turns out that for fixed $\lambda<0$ the local bifurcation diagram near $\mathcal{R}_{0}$ and $\mathcal{R}_{ \pm}$ is determined in a unique way by the total number of singularities. Moreover if $\gamma \geq$ $2 \sqrt{\alpha^{2}+1} / \alpha$, then $\gamma>\lambda+2$; hence, by Propositions 8 and 9, if there appear singularities along $\mathcal{R}_{+}$, then there are always two singularities present along $\mathcal{R}_{0}$. Hence the total number of singularities can be one, two, three, five or seven. Summarized we have the following result.

Proposition 13. Let $\zeta \in \mathcal{P}_{-}$be fixed. Then the number of singularities of (2) is prime and $1 \leq n \leq 7$. The local phase portrait of $Y_{\zeta}$ near the rays $\mathcal{R}_{0}$ and $\mathcal{R}_{ \pm}$is uniquely determined by the number of singularities $n$, and therefore denoted by $\bar{n}$. The slice of the bifurcation diagram of the local phase portraits of $Y_{\zeta}$ near $\mathcal{R}_{0}$ and $\mathcal{R}_{ \pm}$is shown in Figure 2.

In Section 6 we will see that the global bifurcation diagram restricted to $\mathcal{P}_{-}$is completely and uniquely determined by the local one. In Section 7 it becomes clear that this is not the case for $\mathcal{P}_{+}$. The following proposition describes the local bifurcation diagram in this region in terms of the number of singularities.

Proposition 14. The slice of the local bifurcation diagram for $\lambda>0$ fixed is shown in Figure 7. Here we use the following notation to distinguish between different configurations of singularities: $n_{h}, n_{h}^{\mathcal{G}, k}$ or $n_{h}^{k}, 1 \leq n \leq 7, k \in\{l, \mathcal{H}, r\}$ and $h \in\{d, c, u\}$. If $Y_{\zeta}$ has $n$ singularities, the parameter $\zeta$ induces locally near the rays $\mathcal{R}_{0}$ and $\mathcal{R}_{ \pm}$the phase portrait $n_{h}, n_{h}^{\mathcal{G}, k}$ or $n_{h}^{k}$. In case all singularities are on the horizontal axis, $\zeta$ belongs to $n_{h}$; in case that the rays $\mathcal{R}_{ \pm}$each carry one singularity (resp. two singularities), $\zeta$ belongs to $n_{h}^{\mathcal{G}, k}$ (resp. $n_{h}^{k}$ ). Furthermore the configuration of singularities along $\mathcal{R}_{0}$ is specified by the lower index $h$ according to the following scheme:

\begin{tabular}{c|cc}
$h$ & ${ }_{h}$ & $\mathcal{R}_{0}$ \\
\hline$u$ & $\zeta \in \mathcal{F} \cup \mathcal{F}_{u}$ & one or two \\
$c$ & $\zeta \in \mathcal{F}_{d} \cap \mathcal{E}_{u}$ & zero \\
$d$ & $\zeta \in \mathcal{E} \cup \mathcal{E}_{d}$ & one or two
\end{tabular}

The configuration of the singularities along $\mathcal{R}_{ \pm}$is specified by the upper index, which is of type ${ }^{\mathcal{G}, k}$ or ${ }^{k}$ depending whether it has one resp. two singularities along $\mathcal{R}_{ \pm}$, according to the following scheme:

\begin{tabular}{c|cc||c|cc}
$k$ & $\mathcal{G}, k$ & $\mathcal{R}_{ \pm}$ & $k$ &.${ }^{k}$ & $\mathcal{R}_{ \pm}$ \\
\hline$l$ & $\zeta \in \mathcal{H}_{l} \cap \mathcal{G}$ & one & $l$ & $\zeta \in \mathcal{H}_{l} \cap \mathcal{G}_{r}$ & two \\
$\mathcal{H}$ & $\zeta \in \mathcal{H} \cap \mathcal{G}$ & one & $\mathcal{H}$ & $\zeta \in \mathcal{H} \cap \mathcal{G}_{r}$ & two \\
$r$ & $\zeta \in \mathcal{H}_{r} \cap \mathcal{G}$ & one & $r$ & $\zeta \in \mathcal{H}_{r} \cap \mathcal{G}_{r}$ & two
\end{tabular}

Proof. By Propositions 8, 9, 10 and 11 and Figures 4 and 6, the topological type of the singularities of $Y_{\zeta}$ along $\mathcal{R}_{0}$ and $\mathcal{R}_{ \pm}$and its corresponding number are precisely determined by the notation introduced in previous proposition, and so also the local bifurcation diagram of $Y_{\zeta}$ near $\mathcal{R}_{0}$ and $\mathcal{R}_{ \pm}$. 

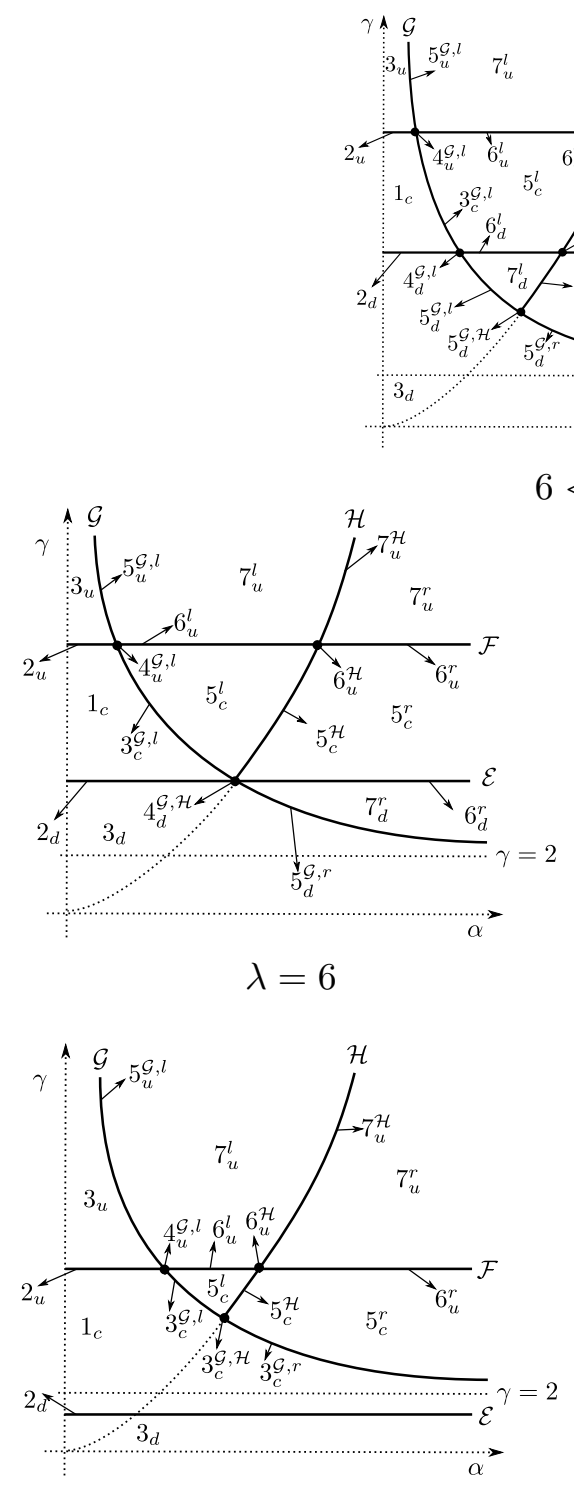

$$
2<\lambda \leq 4
$$

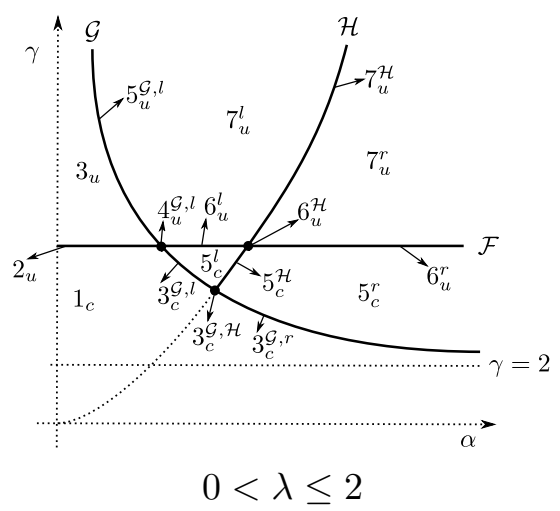

FiguRE 7. Characteristic slices of the local bifurcation diagram for fixed $\lambda>0$.

\section{Periodic, homoclinic And heteroclinic orbits}

For fixed $\zeta$ the divergence is $\operatorname{div}\left(Y_{\zeta}\right)=\operatorname{div}\left(Y_{\zeta}\right)(x, y)=\left(2 \alpha^{2} \lambda-\gamma\right) y$. Hence by Dulac's Theorem (see [10]) we can draw the following conclusion.

Proposition 15. If $\zeta \in \mathcal{P} \backslash \mathcal{H}$, then periodic, homoclinic or heteroclinic orbits intersect the horizontal axis. Furthermore all periodic orbits are part of a continuum.

As a corollary and since $\mathcal{H}$ represents the Hamiltonian vector fields, there are no limit cycles for $Y_{\zeta}$. Furthermore,

Corollary 16. None of the differential systems (1), (2) nor (5) have limit cycles. 


\section{Global Bifurcation diagram in $\mathcal{H} \cap \mathcal{P}$}

By rather straightforward algebraic manipulations we obtain from [8] the topological classification of the Hamiltonian reversible vector fields in (2), i.e. with $\gamma=2 \alpha^{2} \lambda$. Then the Hamiltonian is given by

$$
H_{(\alpha, \lambda)}(x, y)=\frac{1}{2}\left(x^{2}+y^{2}\right)+\frac{1}{3}\left(2 \alpha^{2}-1\right) \lambda x^{3}+\alpha^{2} \lambda x y^{2}+\frac{1}{4}\left(x^{2}+y^{2}\right)^{2} .
$$

In [8] they are represented by (5) with $\xi=0$. Here we add to the result in [8] the global bifurcation diagram.

Theorem 17. Up to topological equivalence the Hamiltonian reversible vector fields of (1) or (2) are given by the 2-parameter family $Y_{\zeta}$ with $\gamma=2 \alpha^{2} \lambda, \alpha>0, \lambda>0$ (i.e $\zeta \in \mathcal{H} \cap \mathcal{P}$ ) and show 14 topologically different phase portraits depending on the parameter $(\alpha, \lambda)$ as shown in Figure 8. The classification for topological equivalence is listed according to the number of singularities as follows: $1_{c} ; 2_{d} ; 3_{d}$ if the singularities are collinear and $3_{c}^{\mathcal{G}, \mathcal{H}}$ if the singularities are noncollinear; $4_{d}^{\mathcal{G}, \mathcal{H}} ; 5_{c}^{\mathcal{H}}$ and $5_{d}^{\mathcal{G}, \mathcal{H}}$ if there are one resp. three singularities on the line of symmetry; $6_{d}^{\mathcal{H}}$ or $6_{u}^{\mathcal{H}}$ that are topologically equivalent; $7_{d}^{\mathcal{H}^{\sigma}}$ if $2 \alpha^{2}-1<0$ and $7_{u}^{\mathcal{H}^{\sigma}}$ if $2 \alpha^{2}-1>0$, where $\sigma=\operatorname{sgn}\left\{\left(2 \alpha^{2}-1\right) \Psi(\alpha, \lambda)\right\}$ if $\Psi(\alpha, \lambda) \neq 0$ and $\sigma=0$ if $\Psi(\alpha, \lambda)=0$ and $\Psi$ reads as

$$
\begin{aligned}
\Psi(\alpha, \lambda)= & \left(2 \alpha^{2}-1\right)\left(\alpha^{2}+1\right)^{2} \sqrt{\left(\left(2 \alpha^{2}-1\right)^{2} \lambda^{2}-4\right)^{3}}+16 \alpha^{3} \sqrt{\left(\alpha^{6} \lambda^{2}-\left(\alpha^{2}+1\right)\right)^{3}} \\
& +8 \alpha^{6} \lambda^{2}-6 \alpha^{2}-3 \lambda^{2} \alpha^{2}+\lambda^{2}-6 .
\end{aligned}
$$

The bifurcation diagram is sketched in Figure 9.
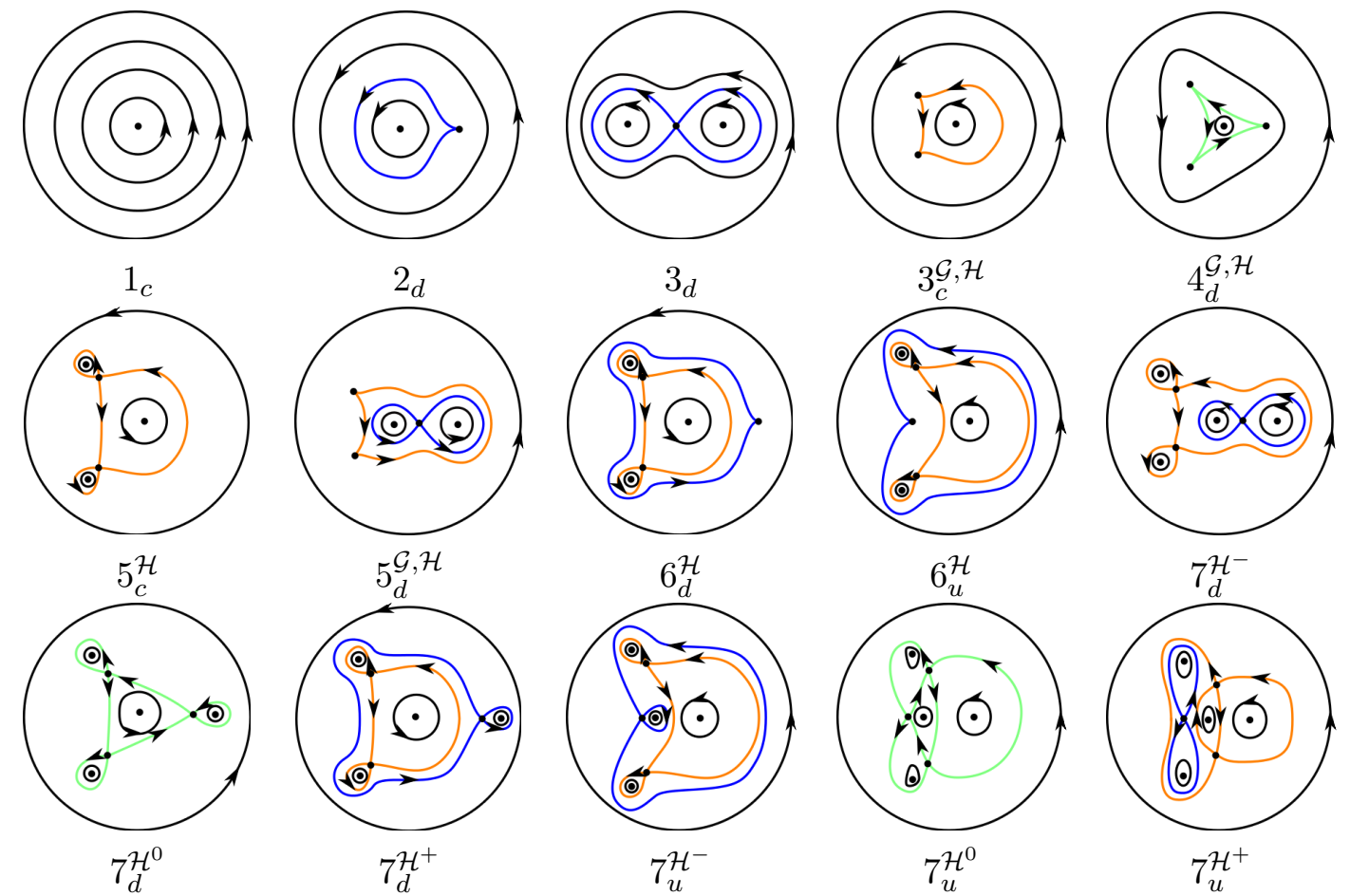

FiguRE 8. Phase portraits in $\mathcal{P} \cap \mathcal{H}$. 
In Figure 8 it is seen that the phase portraits $6_{d}^{\mathcal{H}}$ and $6_{u}^{\mathcal{H}}$ are topologically equivalent; both representations are included to visualize better the bifurcation that happens when passing through the bifurcation curves in Figure 9.

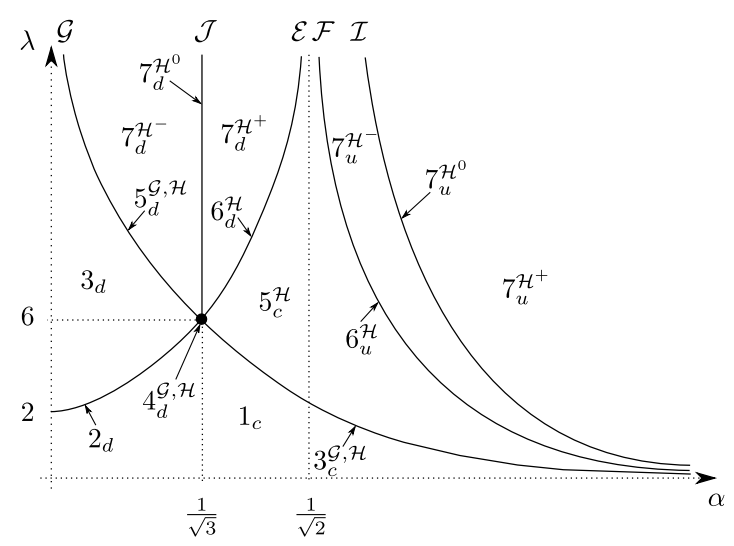

FiguRE 9. Global bifurcation diagram in $\mathcal{P} \cap \mathcal{H}$, projected in the $(\alpha, \lambda)$ plane, see Theorem 17 and Figure 8.

Let us recall some notations used in Theorem 17 and Figure 9. The curves labeled by $\mathcal{E}, \mathcal{F}, \mathcal{G}$ correspond to the local bifurcations defined in Section 3.3, intersected with $\gamma=2 \alpha^{2} \lambda$ and projected in the $(\alpha, \lambda)$-plane. The curves labeled by $\mathcal{I}, \mathcal{J}$ correspond to global bifurcations, more precisely the ones of crossing of separatrices. These only appear when there are seven singularities. In particular, for the Hamiltonian case, when the local phase portrait is given by $7_{u}^{\mathcal{H}}$ or $7_{d}^{\mathcal{H}}$ respectively. The bifurcation surfaces $\mathcal{I}$ and $\mathcal{J}$ restricted to $\mathcal{H}$ distinguish between 3 possible global phase portraits $7_{u}^{\mathcal{H}^{\sigma}}$ and $7_{d}^{\mathcal{H}^{\sigma}}$ respectively, where $\sigma \in\{-, 0,+\}$. From [8] it is deduced that $\mathcal{I}$ restricted to $\mathcal{H}$ is the semi-algebraic set $\Psi^{-1}(0) \cap \mathcal{P} \cap \mathcal{H} \cap \mathcal{F}_{u}$, where $\Psi$ is defined in (27). The curve $\mathcal{J}$ restricted to $\mathcal{H}$ is the semi-algebraic set $\left\{(\alpha, \lambda) \in \mathbb{R}^{2}: \alpha=1 / \sqrt{3}, \lambda \geq 6\right\}$.

To end we draw the global bifurcation diagram of Hamiltonian reversible vector fields for (5), i.e. with $\xi=0$, to complete the result in [8], for $g>0, e \in \mathbb{R}$. Notice that the family $Y_{\left(\alpha, 2 \alpha \lambda^{2}, \lambda\right)}, \alpha, \lambda>0$ is a strict subfamily of $X_{(g, 0, e)}^{R}, g<0, e \in \mathbb{R}$, that corresponds with $e<2 g, g>0$. Although for topological equivalence no other phase portraits are found than the ones presented in Figure 8, there is a new bifurcation curve that is denoted by $\mathcal{D}$. Along $\mathcal{D}$ the ray $\mathcal{R}_{0}$ is triple (i.e. $A(0)=A^{\prime}(0)=A^{\prime \prime}(0)=0, A^{\prime \prime \prime}(0) \neq 0$ ) implying that the singularities along $\mathcal{R}_{0}$ are non-elementary. To highlight this fact the corresponding phase portraits are denoted by $2_{t}$ and $3_{t}$ for the case of two and three singularities respectively, and are topologically equivalent to $2_{d}$ and $3_{d}$ respectively (see Figure 11). In particular the bifurcation diagram for the Hamiltonian reversible phase portraits of $(5)$ covers the parameter plane $\pi_{1}(\mathcal{Q}(\mathcal{H} \cap \mathcal{P})) \cup\{(g, e): e \geq 2 g, g>0\}$, as in Figure 10 that thus can be obtained by transformation of Figure 9 by $\mathcal{Q}$, given in (6), and adding the region $e \geq 2 g, g>0$ (including the bifurcation curve $\mathcal{D}$ ).

The transformed bifurcation curves in Figure 10 are called correspondingly again by $\mathcal{E}, \mathcal{F}, \mathcal{G}, \mathcal{I}, \mathcal{J}$. Now the algebraic set $\mathcal{I}$ restricted to $\mathcal{H}$ is determined by $\psi$, with

$$
\begin{aligned}
\psi(g, e)= & 2304 g^{2}-1536 g e+256 e^{2}-1152 g^{4}+384 g^{3} e-864 g^{2} e^{2}+576 g e^{3}-96 e^{4}+144 g^{6} \\
& +360 g^{4} e^{2}-120 g^{3} e^{3}+81 g^{2} e^{4}-54 g e^{5}+9 e^{6}-32 g^{6} e^{2}-24 g^{4} e^{4}+8 g^{3} e^{5},
\end{aligned}
$$

and $\mathcal{J}$ restricted to $\mathcal{H}$ corresponds to the semi-algebraic set $\{(g, e): g>2, e+g=0\}$. 


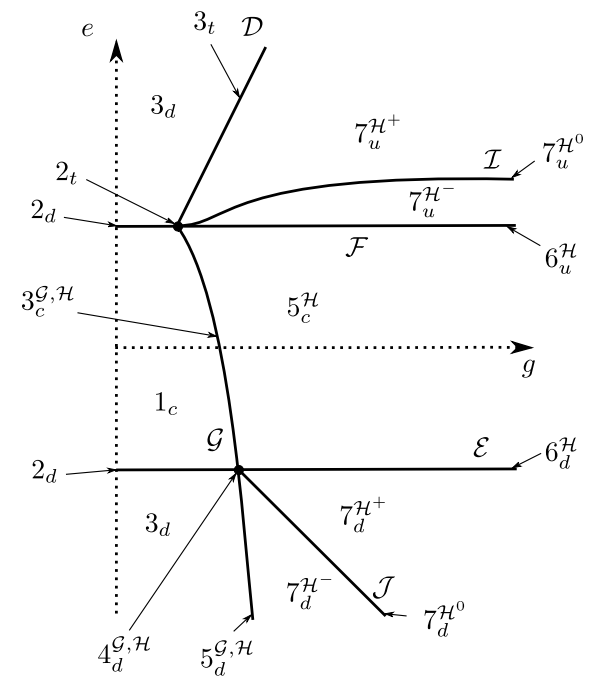

FiguRE 10. Global bifurcation diagram of $X_{(g, 0, e)}^{R}$, for $g>0, e \in \mathbb{R}$.

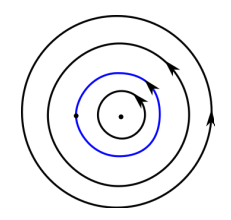

$2 t$

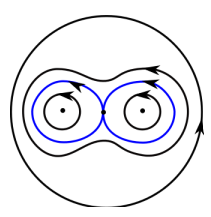

$3_{t}$

Figure 11. Phase portraits of $X_{(g, 0, e)}^{R}$ for $e=2 g, g>0$, see Figure 10.

\section{Global Bifurcation diagram in $\mathcal{P}_{-}$}

In this section we prove Theorem 4 establishing that for $\lambda<0$ the local phase portraits $\bar{n}$ (where $n=1,2,3,5,7)$ near $\mathcal{R}_{0}$ and $\mathcal{R}_{ \pm}$in Proposition 13 determine uniquely the global phase portraits. Therefore we use the same notation to refer to the global phase portraits as to refer to the local ones. As a consequence the global phase portraits can be distinguished by the total number of singularities.

To this end we start by a technical lemma. As in Propositions 10 and 11 we use the notations $\mathbf{s}_{ \pm}^{1}=\left(x_{ \pm}^{1}, y_{ \pm}^{1}\right)$ for the saddle and $\mathbf{s}^{1}=\left(x^{1}, y^{1}\right)$ for the saddle-node along $\mathcal{R}_{+}$.

Lemma 18. Let $\zeta \in\left(\mathcal{G} \cup \mathcal{G}_{r}\right) \cap \mathcal{P}_{-}$. Then the relative position of the singularities and the direction of the flow of $Y_{\zeta}$ in $\{y \geq 0\}$ is as indicated in Figure 12. More precisely, for $\zeta \in \mathcal{G}_{r}$ :

(1) The vertical line $x=x_{+}^{1}$ intersects the segment bounded by the center point $\left(x_{+}^{0}, 0\right)$ and the saddle point $\left(x_{-}^{0}, 0\right)$, i.e., $x_{+}^{0}<x_{+}^{1}<x_{-}^{0}$.

(2) The direction of the flow of the vector field $Y_{\zeta}$ along the vertical $x=x_{+}^{1}$ is as follows:

$$
\begin{aligned}
& \left.\dot{x}\right|_{x=x_{+}^{1}, 0<y<y_{+}^{1}}>0,\left.\dot{y}\right|_{x=x_{+}^{1}, 0<y<y_{+}^{1}}>0, \\
& \left.\dot{x}\right|_{x=x_{+}^{1}, y>y_{+}^{1}}<0 \text { and }\left.\dot{y}\right|_{x=x_{+}^{1}, y>y_{+}^{1}}<0 .
\end{aligned}
$$


These properties are sketched in Figure 12(a). By reflection about the $x$-axis the behavior in $\{y<0\}$ is obtained. Analogous properties hold for $\zeta \in \mathcal{G}$, only replacing $x_{+}^{1}$ and $y_{+}^{1}$ by $x^{1}$ and $y^{1}$ respectively, and are sketched in Figure 12(b).

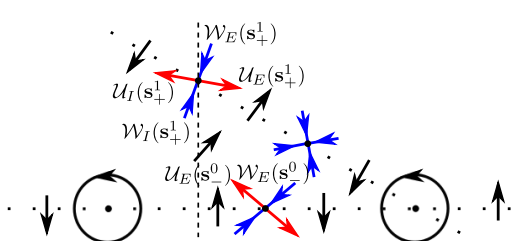

(a)

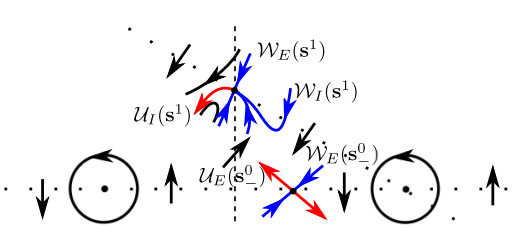

(b)

FiguRE 12. Local phase portrait of $Y_{\zeta}$ in $\{y \geq 0\}$ for (a) $\mathcal{G}_{r} \cap \mathcal{P}_{-}$and (b) $\mathcal{G} \cap \mathcal{P}_{-}$, see Lemma 18 .

Proof. The claims follow by direct algebraic manipulation using the expression in (20). Notice furthermore that $\left|x_{+}^{0}\right|>1 \geq\left\|\left(x_{+}^{1}, y_{+}^{1}\right)\right\|>\left|x_{+}^{1}\right|$ and that $\left|x_{-}^{0}\right|>\left|x_{+}^{1}\right|$ if and only if

$$
\left(x_{+}^{1}\right)^{2}+\left(y_{+}^{1}\right)^{2}+1+\lambda x_{+}^{1}+\sqrt{\left(\left(x_{+}^{1}\right)^{2}+\left(y_{+}^{1}\right)^{2}+1+\lambda x_{+}^{1}\right)^{2}-4\left(x_{+}^{1}\right)^{2}}<2\left(x_{+}^{1}\right)^{2} .
$$

This last inequality holds for $\left(y_{+}^{1}\right)^{2}+\lambda x_{+}^{1}>0$. To obtain the signs in (28), one calculates

$$
\begin{aligned}
& \left.\dot{x}\right|_{x=x_{+}^{1}}=y\left(y_{+}^{1}-y\right)\left(y_{+}^{1}+y\right), \\
& \left.\dot{y}\right|_{x=x_{+}^{1}}=-x_{+}^{1}\left(y_{+}^{1}-y\right)\left(y_{+}^{1}+y\right)\left(\left(y_{+}^{1}\right)^{2}+\lambda x_{+}^{1}\right) /\left(y_{+}^{1}\right)^{2} .
\end{aligned}
$$

Recall that the rays $\mathcal{R}_{0}$ and $\mathcal{R}_{ \pm}$are isoclines for $\dot{r}=0$.

Proof of Theorem 4. The global phase portraits $\overline{1}, \overline{2}$, and $\overline{3}$ are obtained directly from the local phase portraits described in Propositions 8 and 13 and Figure 3 and clearly are uniquely determined. Using Lemma 18, Figure 12 and the Poincaré-Bendixson Theorem we find that the relative positions of the separatrices in $\{y \geq 0\}$ in cases $\overline{5}$ and $\overline{7}$ are as pictured in Figures 13 and 14 respectively. Using the reversibility property one obtains that the global phase portraits in cases $\overline{5}$ and $\overline{7}$ are uniquely determined and as drawn in Figure 1.

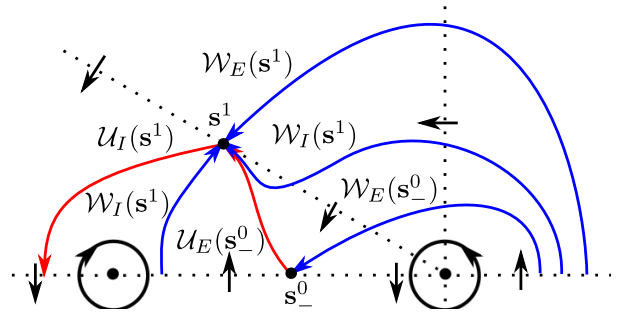

Figure 13. Relative positions of the separatrices in $\{y \geq 0\}$ in case $\overline{5}$.

The proof that the relative positions of the separatrices in case $\overline{5}$ are as drawn in Figure 13, is analogous to the one for Figure 14 in case $\overline{7}$. We only detail this last case.

By Lemma 18, in backward time, the separatrix $\mathcal{W}_{I}\left(\mathbf{s}_{+}^{1}\right)$ has to intersect the horizontal axis in between $x_{+}^{0}$ and $x_{+}^{1}$. Next $\mathcal{U}_{I}\left(\mathbf{s}_{+}^{1}\right)$ in forward time has to intersect the horizontal 


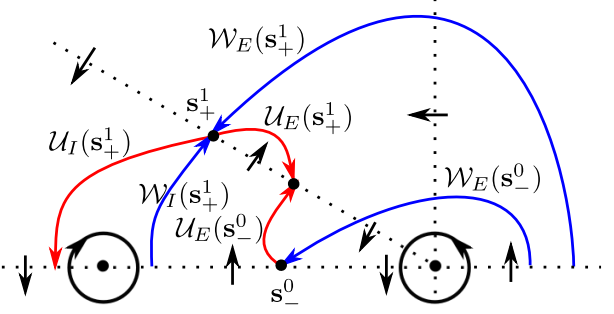

Figure 14. Relative positions of the separatrices in $\{y \geq 0\}$ in case $\overline{7}$.

axis on the left of $x_{+}^{0}$. By the direction of the flow along $\mathcal{R}_{+}$between $\mathbf{s}_{-}^{1}$ and $\mathbf{s}_{+}^{1}$ and the Poincaré-Bendixson Theorem, the separatrix $\mathcal{U}_{E}\left(\mathbf{s}_{+}^{1}\right)$ has to connect the singularities along $\mathcal{R}_{+}$like pictured in Figure 14. Furthermore, in backward time, $\mathcal{W}_{E}\left(\mathbf{s}_{+}^{1}\right)$ has to intersect the positive horizontal axis. By Poincaré-Bendixson Theorem, $\mathcal{U}_{E}\left(\mathbf{s}_{-}^{0}\right)$ has to connect the singularities $\mathbf{s}_{-}^{0}$ and $\mathbf{s}_{-}^{1}$, and the separatrix $\mathcal{W}_{E}\left(\mathbf{s}_{-}^{0}\right)$ has to intersect the positive horizontal axis on the left of the intersection $\mathcal{W}_{E}\left(\mathbf{s}_{+}^{1}\right) \cap\{y=0\}$. Therefore the relative positions of the separatrices in $\{y \geq 0\}$ is uniquely determined as in Figure 14, ending the proof.

\section{Global phase portraits in $\mathcal{P}_{+}$}

The global bifurcation diagram for fixed $\lambda>0$ is obtained by the local one as described in Proposition 14 and shown in Figure 7, adding the global bifurcation curves that determine the crossing of separatrices.

In this case there are a lot more possibilities even for the local phase portrait than in the case treated in Section 6. For one, two and three singularities the local phase portrait near $\mathcal{R}_{0}$ and $\mathcal{R}_{ \pm}$completely determines the global one, see Proposition 19 . The other cases are subsequently analyzed in Sections 7.2 until 7.5 starting with the case of the maximal number of singularities. In particular, in Section 7.2, the classification for this case is proven and summarized in Proposition 20. Notice that in this case all singularities are non-degenerate and elementary. This is also the case for five singularities if all of them different from the origin are along $\mathcal{R}_{ \pm}$. However most often this is not the case and there are singularities along $\mathcal{R}_{0}$ as well as along $\mathcal{R}_{ \pm}$; then the phase portrait is obtained from the case with seven singularities by coalescence of a saddle and a node to a saddle-node on both rays $\mathcal{R}_{ \pm}$. Both cases with five singularities are treated in Section 7.3. Next, in Section 7.4 (resp. 7.5) the case of six (resp. four) singularities is obtained as continuous bifurcation from the case with seven to five (resp. five to three and six to two) singularities. In Section 7.5 we also add a detailed numerical study that completes the case of four singularities. Finally in Section 7.6 we draw the global bifurcation diagram with the partial help of numerical analysis.

From the classification results in this section, i.e. Propositions 19, 20, 21, 22 and 23, we find the classification of the phase portraits in $\mathcal{P}_{+}$, that is summarized in Theorem 5 .

7.1. Less than or equal to three singularities. The following proposition describes the classification of the phase portraits with at most three singularities and is obtained by using the symmetry property and the local classification from Section 3. 
Proposition 19. Let $\zeta \in \mathcal{P}_{+}$. The phase portrait of (2) has at most three singularities if and only if $\zeta \in \mathcal{G}_{l} \cup\left(\mathcal{G} \cap \mathcal{E}_{u} \cap \mathcal{F}_{d}\right)$. Furthermore the global phase portrait is uniquely determined by the local phase portrait and there are exactly 6 topologically different ones, that are drawn in Figure 15 and are listed by increasing number of singularities: $1_{c}$ in $\mathcal{G}_{l} \cap \mathcal{E}_{u} \cap \mathcal{F}_{d} ; 2_{d}$ in $\mathcal{G}_{l} \cap \mathcal{E}$ or $2_{u}$ in $\mathcal{G}_{l} \cap \mathcal{F}$ (these are equivalent); $3_{d}$ in $\mathcal{G}_{l} \cap \mathcal{E}_{d}, 3_{u}$ in $\mathcal{G}_{l} \cap \mathcal{F}_{u}, 3_{c}^{\mathcal{G}, t}$ in $\mathcal{G} \cap \mathcal{H}_{t}$ (where $t \in\{l, r\}$, both determining topologically the same phase portrait) and $3_{c}^{\mathcal{G}, \mathcal{H}}$ in $\mathcal{G} \cap \mathcal{H}$.
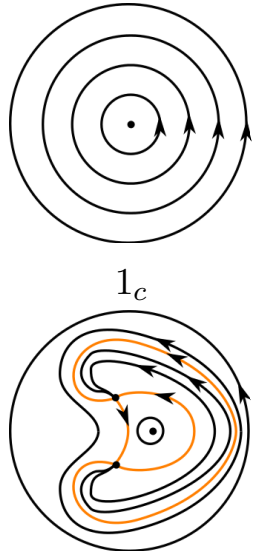

$3_{c}^{\mathcal{G}, l}$
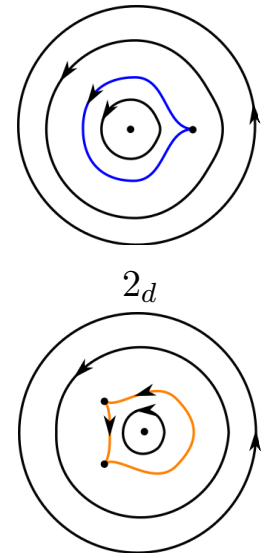

$3_{c}^{\mathcal{G}, \mathcal{H}}$
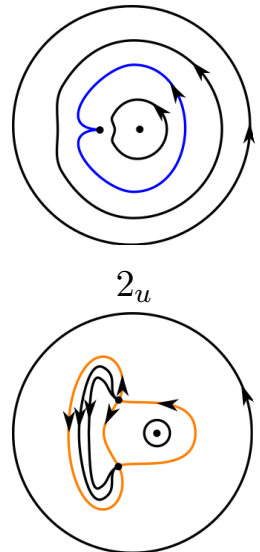

$3_{c}^{\mathcal{G}, r}$
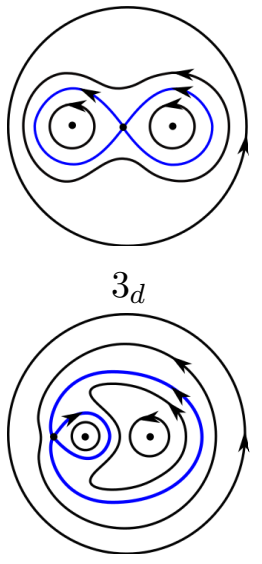

$3_{u}$

FiguRE 15. Phase portraits of $Y_{\zeta}$ with one, two or three singularities for $\zeta \in \mathcal{P}_{+}$, see Proposition 19.

\subsection{Seven singularities.}

Proposition 20. Let $\zeta \in \mathcal{P}_{+}$. The phase portrait of (2) has seven singularities if and only if $\zeta \in\left(\mathcal{E}_{d} \cup \mathcal{F}_{u}\right) \cap \mathcal{G}_{r}$. In this case there are 20 topologically different phase portraits. More precisely, there are 9 (resp. 11) in $\mathcal{E}_{d} \cap \mathcal{G}_{r}$ (resp. $\mathcal{F}_{u} \cap \mathcal{G}_{r}$ ) that are listed in Figure 16 (resp. Figure 17) and all together they are shortly referred to as $7_{d}$ (resp. $7_{u}$ ). In particular, $7_{z}^{l^{\sigma}}$ and $7_{z}^{r^{\sigma}}$ with $\sigma \in\{+,-\}$ are equivalent, for $z=d$ (resp. $z=u$ ).

Notice that the specific composition of the phase portraits in Figures 16 and 17 respects the subsequent bifurcation phenomena that happen between adjacent phase portraits and is detailed at the end of this section. This also is the reason why some topologically equivalent phase portraits appear.

Proof of Proposition 20. From the local bifurcation diagram in Figure 7 and Proposition 14 we know that the case with seven singularities distinguishes between $7_{d}$ and $7_{u}$. To complete the global phase portraits we analyze the separatrices at the saddles, and follow the flux forward and backward in time. By the reversibility property we can restrict our attention to the half plane $\{y \geq 0\}$.

We only detail the proof of case $7_{d}$. The possible intersections of $\mathcal{W}_{I}\left(\mathbf{s}_{-}^{1}\right)$ and $\mathcal{U}_{E}\left(\mathbf{s}_{-}^{1}\right)$ with the ray $\mathcal{R}_{+}$in backward resp. forward time are shown in Figure 18. The case (b) corresponds to the Hamiltonian one. The possibilities (a), (b), (c) are referred to as $7_{d}^{r}, 7_{d}^{\mathcal{H}}, 7_{d}^{l}$ respectively. Next for each of these cases we analyze the possible intersections of $\mathcal{W}_{E}\left(\mathbf{s}_{-}^{1}\right)$ and $\mathcal{U}_{E}\left(\mathbf{s}_{-}^{0}\right)$ with the vertical axis in backward resp. forward time; for further 


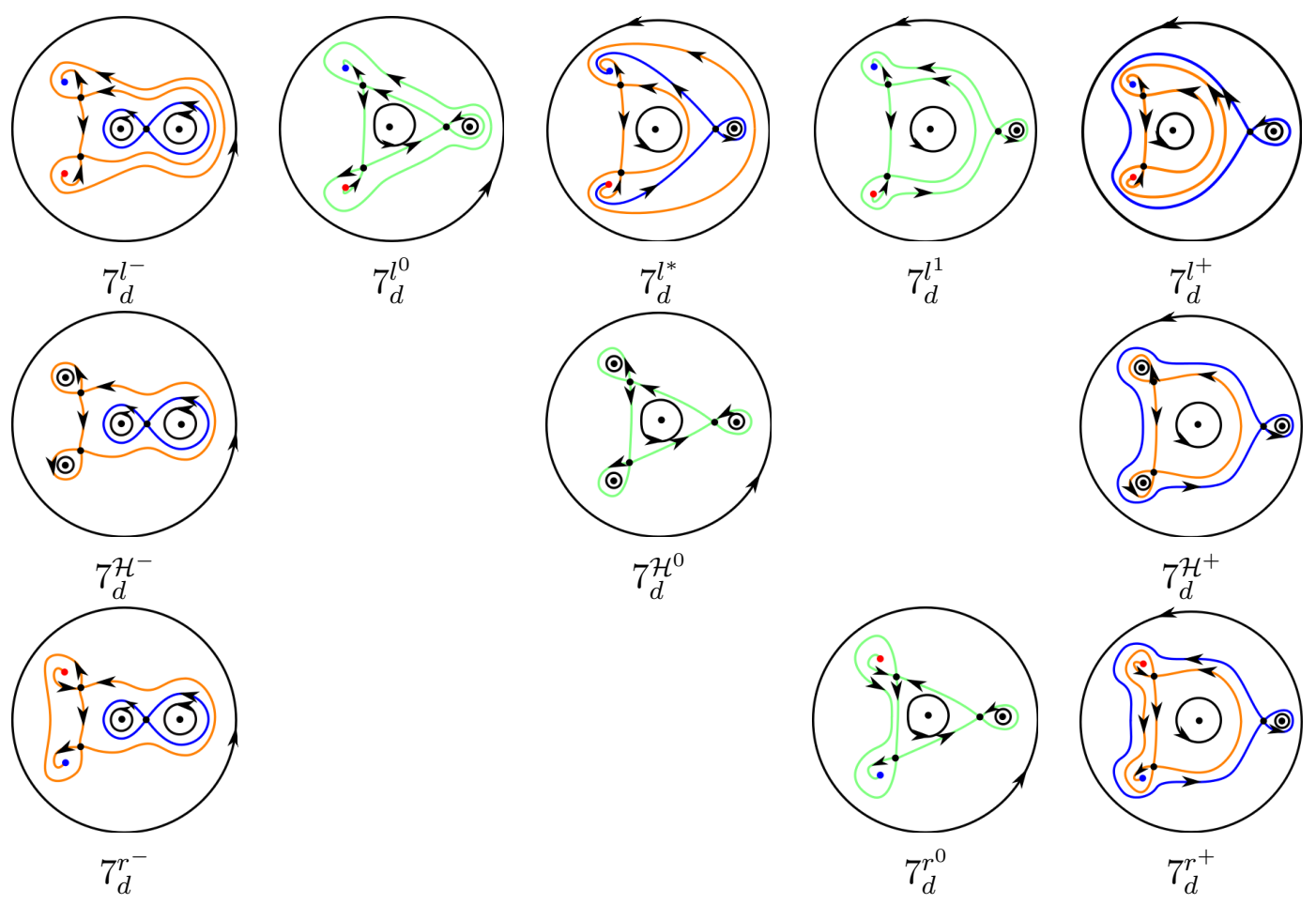

Figure 16. Phase portraits in $\mathcal{P}_{+} \cap \mathcal{G}_{r} \cap \mathcal{E}_{d}$ (referred to as $7_{d}$ ), see Proposition 20.
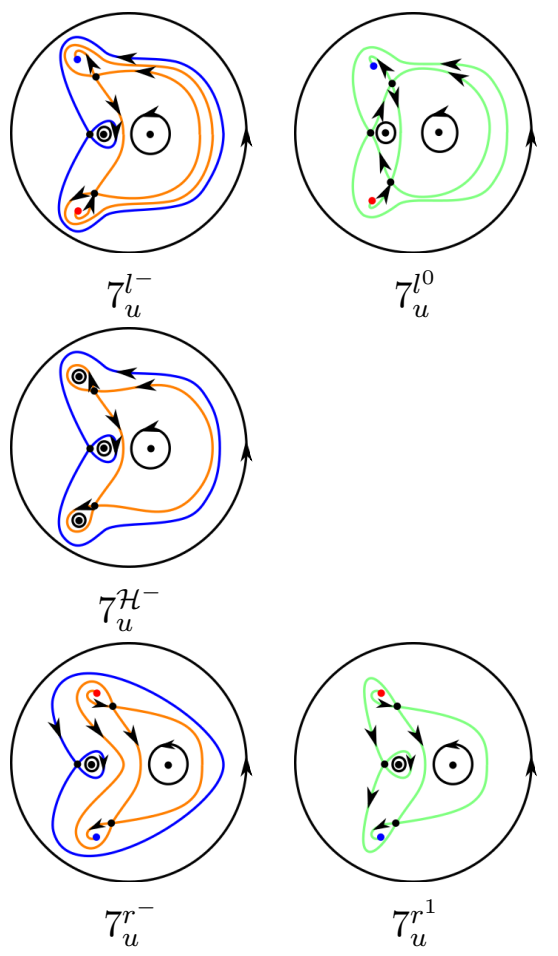
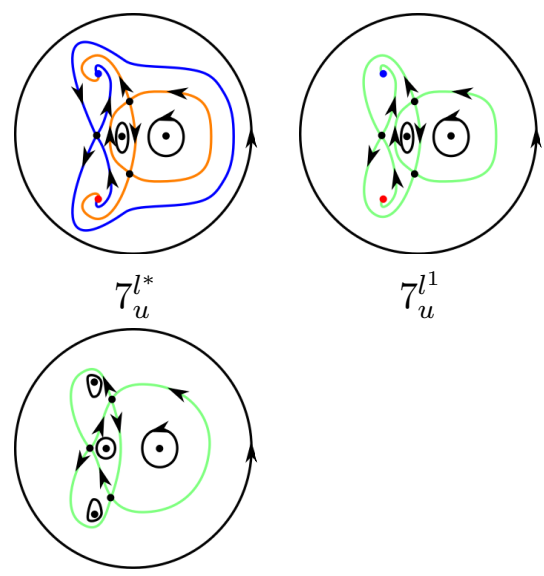

$7_{u}^{l^{1}}$
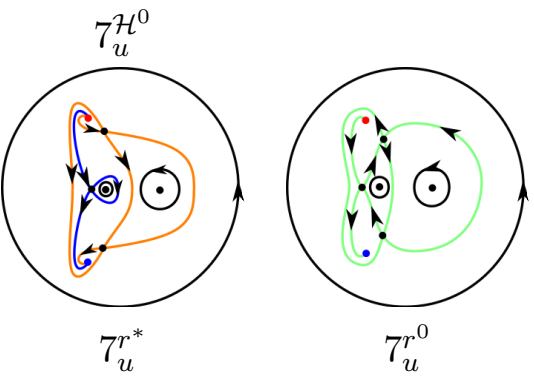
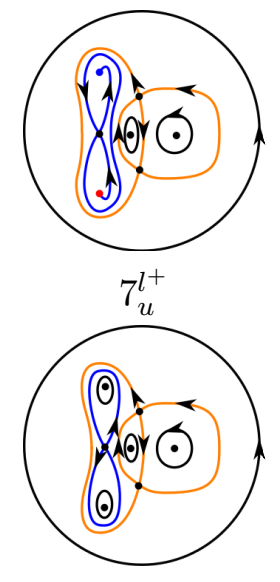

Figure 17. Phase portraits in $\mathcal{P}_{+} \cap \mathcal{G}_{r} \cap \mathcal{F}_{u}$ (referred to as $7_{u}$ ), see Proposition 20 . 
reference the $y$-coordinates are denoted by $y_{E}^{1}$ and $y_{E}^{0}$. Additionally in case (c) we analyze the possible intersections of $\mathcal{W}_{I}\left(\mathbf{s}_{-}^{1}\right)$ and $\mathcal{U}_{E}\left(\mathbf{s}_{-}^{0}\right)$ with the vertical axis in backward resp. forward time; as before the $y$-coordinates are denoted by $y_{I}^{1}$ and $y_{E}^{0}$. Observe that this leads to 3 possibilities in cases (a) as well as (b), and 5 possibilities in case (c), adding to 11 possibilities in total for case $7_{d}$. Then clearly always $y_{E}^{1}<y_{I}^{1}$ (if it exists) and so all the possibilities are summarized by:

(a) (b) (c)

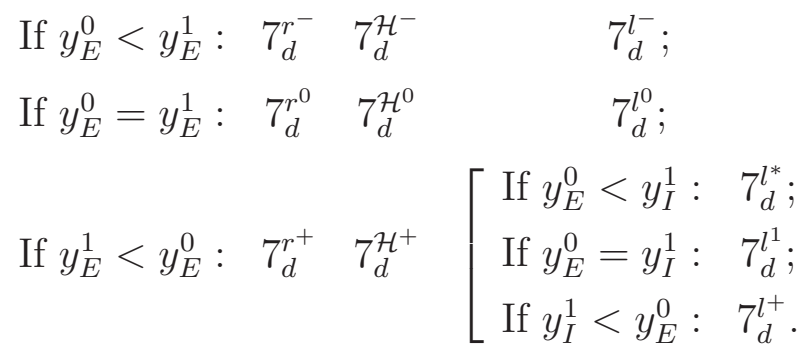

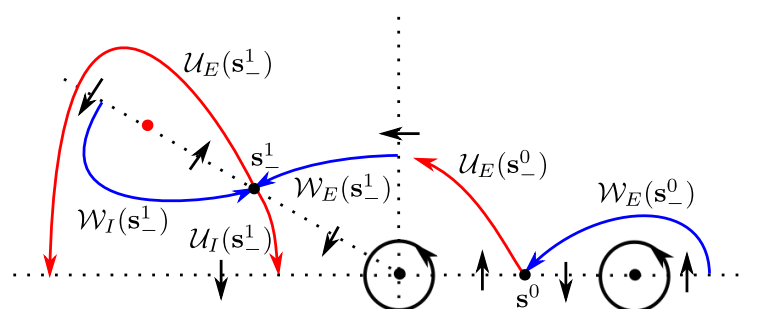

(a)

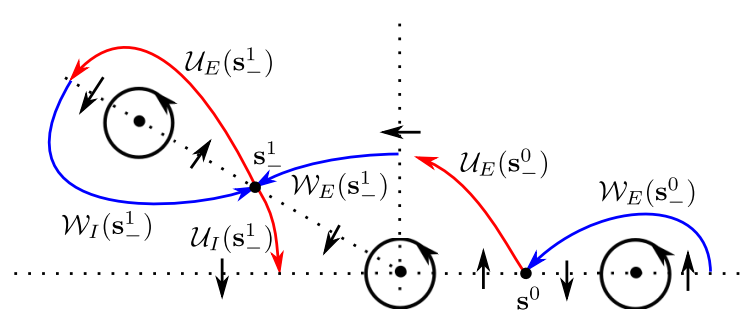

(b)

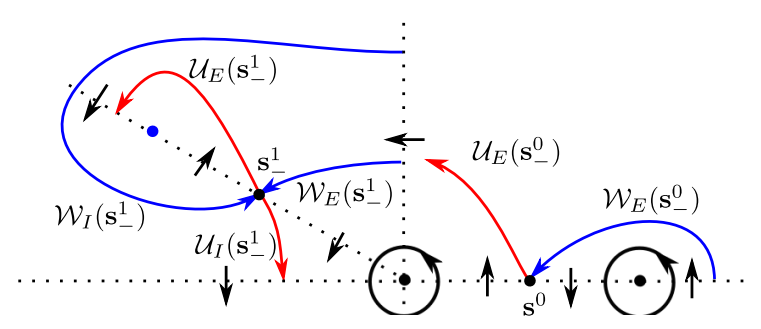

(c)

Figure 18. Possibilities for the separatrices in case $7_{d}$.

The cases $7_{d}^{z^{0}}$, where $z \in\{r, \mathcal{H}, l\}$, correspond to the fact that $\mathcal{W}_{E}\left(\mathbf{s}_{-}^{1}\right)=\mathcal{U}_{E}\left(\mathbf{s}_{-}^{0}\right)$. The case $7_{d}^{l^{1}}$ corresponds to the fact that $\mathcal{W}_{I}\left(\mathbf{s}_{-}^{1}\right)=\mathcal{U}_{E}\left(\mathbf{s}_{-}^{0}\right)$.

We now show that all these possibilities occur and correspond to the phase portraits as drawn in Figure 16. The existence of the Hamiltonian phase portraits, i.e. case (b), 
follows from [8]. In all these cases the separatrices $\mathcal{W}_{I}\left(\mathbf{s}_{-}^{1}\right)$ and $\mathcal{U}_{E}\left(\mathbf{s}_{-}^{1}\right)$ coincide. Bifurcating from $7_{d}^{\mathcal{H}^{-}}$or $7_{d}^{\mathcal{H}^{+}}$the only singularity that changes its type is $\mathbf{s}_{+}^{1}$, and changes from center to stable or unstable focus. By continuity, the attracting or repelling nature of $\mathbf{s}_{+}$, Proposition 15 and the reversibility with respect to the horizontal axis, the connection $\mathcal{W}_{I}\left(\mathbf{s}_{-}^{1}\right)=\mathcal{U}_{E}\left(\mathbf{s}_{-}^{1}\right)$ breaks in only one possible way for each of the cases as drawn in Figure 18(a) and (c). Therefore the global phase portraits denoted by $7_{d}^{r^{-}}, 7_{d}^{r^{+}}, 7_{d}^{l^{-}}$ and $7_{d}^{l^{+}}$exist for values of the parameter close to the Hamiltonian case. Next $7_{d}^{r^{0}}$ (resp. $7_{d}^{l^{0}}, 7_{d}^{*^{*}}, 7_{d}^{l^{1}}$ ) exists by continuous dependence on the parameter and the existence of $7_{d}^{r^{-}}$ and $7_{d}^{r^{+}}$(resp. $7_{d}^{l^{-}}$and $7_{d}^{l^{+}}$). This ends the proof of this case.

The case $7_{u}$ is obtained in a similar way as the case $7_{d}$. Then we distinguish the different phase portraits based on the possible intersections of the separatrices at the saddles $\left(\mathbf{s}_{-}^{1}\right.$ and $\left.\mathbf{s}_{+}^{0}\right)$ with the straight line through the anti-saddles $\left(\mathbf{s}_{+}^{1}\right.$ and $\left.\mathbf{s}_{-}^{0}\right)$, see Figure 19. It is seen that $7_{z}^{l^{\sigma}}$ and $7_{z}^{r^{\sigma}}$ with $\sigma \in\{+,-\}$ are equivalent in case $7_{z}$, for

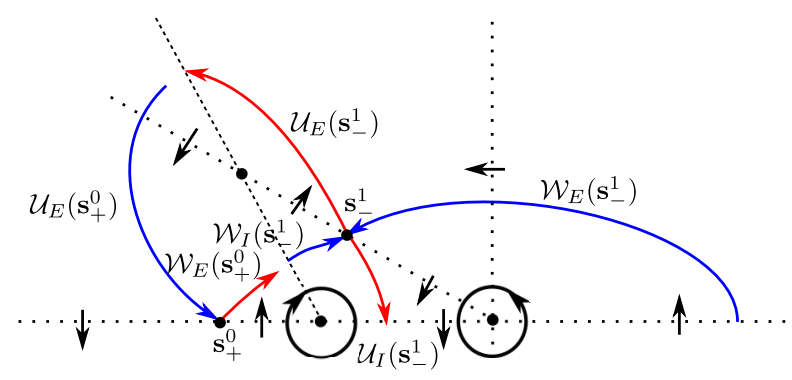

Figure 19. Possibilities for the separatrices in case $7_{u}$.

$z \in\{u, d\}$. Comparing the phase portraits in Figure 16 with the ones in Figure 17, it is found that these phase portraits are mutually different.

From the previous proof we notice that the only 2 possible connections in the nonHamiltonian case $7_{d}$ are found and defined by the bifurcation surfaces:

$$
\mathcal{J}=\left\{\zeta: \mathcal{W}_{E}\left(\mathbf{s}_{-}^{1}\right)=\mathcal{U}_{E}\left(\mathbf{s}_{-}^{0}\right)\right\} \quad \text { and } \quad \mathcal{K}=\left\{\zeta: \mathcal{W}_{I}\left(\mathbf{s}_{-}^{1}\right)=\mathcal{U}_{E}\left(\mathbf{s}_{-}^{0}\right)\right\}
$$

In the non-Hamiltonian case $7_{u}$ the 2 connections are found and defined by the bifurcation surfaces:

$$
\mathcal{I}=\left\{\zeta: \mathcal{W}_{E}\left(\mathbf{s}_{+}^{0}\right)=\mathcal{W}_{I}\left(\mathbf{s}_{-}^{1}\right)\right\} \quad \text { and } \quad \mathcal{L}=\left\{\zeta: \mathcal{U}_{E}\left(\mathbf{s}_{+}^{0}\right)=\mathcal{U}_{E}\left(\mathbf{s}_{-}^{1}\right)\right\} .
$$

In Section 7.6 we draw these surfaces in the bifurcation diagram with the aid of numerical methods.

Notice that the specific composition of the different phase portraits in Figures 16 and 17 is according to the bifurcation surfaces $\mathcal{H}, \mathcal{I}, \mathcal{J}, \mathcal{K}$ and $\mathcal{L}$; the phase portraits on the middle line correspond to $\mathcal{H}$, say the Hamiltonian surface, and the upper resp. lower line correspond to $\mathcal{H}_{l}$ resp. $\mathcal{H}_{r}$. In Figure 16 the diagonal connecting the phase portraits referred to as $7_{d}^{z^{0}}$ with $z \in\{l, \mathcal{H}, r\}$ corresponds to the bifurcation surface $\mathcal{J}$, and the branch from $7_{d}^{\mathcal{H}^{0}}$ to $7_{d}^{l^{1}}$ corresponds to the bifurcation surface $\mathcal{K}$. In Figure 17 the diagonal connecting the phase portraits referred to as $7_{u}^{z^{b}}$ with $z \in\{l, r\}$ and $b=0$ (resp. $b=1$ ) passing through $7_{u}^{\mathcal{H}^{0}}$ corresponds to the bifurcation surface $\mathcal{I}$ (resp. $\mathcal{L}$ ). 
7.3. Five singularities. In case of five singularities there are essentially two cases to be distinguished, whether or not all singularities are non-degenerate and elementary. If it is the case, the singularities different from the origin are located along $\mathcal{R}_{ \pm}$, and the local phase portraits are referred to as $5_{c}$. However, most often this is not the case; then the corresponding parameters belong to $\mathcal{G}$. In Figure 23 the global bifurcation diagram restricted to $\mathcal{G}$ is drawn. Notice that only bifurcation curves are drawn that lead to the lower bound in the classification. Furthermore the dashed lines correspond to the bifurcation curves that are numerically obtained. Next theorem summarizes the topological classification that is found in all cases of five singularities.

Proposition 21. Let $\zeta \in \mathcal{P}_{+}$. The phase portrait of (2) presents five singularities for $\zeta \in\left(\mathcal{G} \cap\left(\mathcal{E}_{d} \cup \mathcal{F}_{u}\right)\right) \cup\left(\mathcal{G}_{r} \cap \mathcal{E}_{u} \cap \mathcal{F}_{d}\right)$. In this case there are $9 \leq n \leq 12$ topologically different phase portraits.

(1) There are exactly 3 in $\mathcal{G} \cap \mathcal{F}_{u}$ as shown in Figure 20 and are referred to as $5_{u}$.

(2) There are exactly 2 in $\mathcal{G}_{r} \cap \mathcal{E}_{u} \cap \mathcal{F}_{d}$. These are shown in Figure 21 and are referred to as $5_{c}$. In particular, $5_{c}^{l}$ and $5_{c}^{r}$ are equivalent.

(3) There are $4 \leq n \leq 7$ in $\mathcal{G} \cap \mathcal{E}_{d}$. More precisely, there are exactly 4 in $\mathcal{G} \cap \mathcal{E}_{d} \cap$ $\left(\mathcal{H}_{r} \cup \mathcal{H}\right)$ and $1 \leq n \leq 5$ in $\mathcal{G} \cap \mathcal{E}_{d} \cap \mathcal{H}_{l}$. These are shown in Figure 22 and are referred to as $5_{d}$. In particular, $5_{d}^{\mathcal{G}, l}$ and $5_{d}^{\mathcal{G}, r^{-}}$are equivalent.
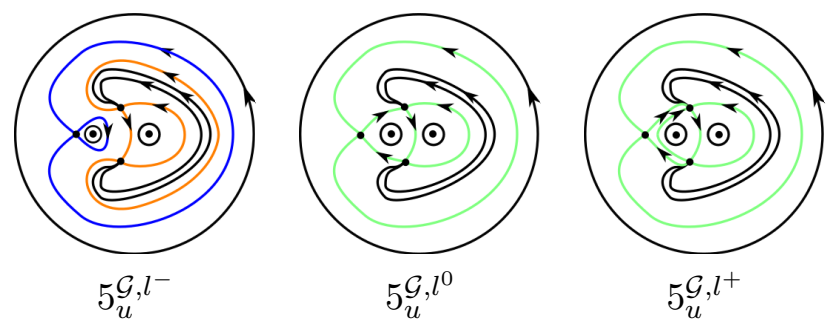

FiguRE 20. Phase portraits in $\mathcal{P}_{+} \cap \mathcal{G} \cap \mathcal{F}_{u}$ (referred to as $5_{u}$ ).
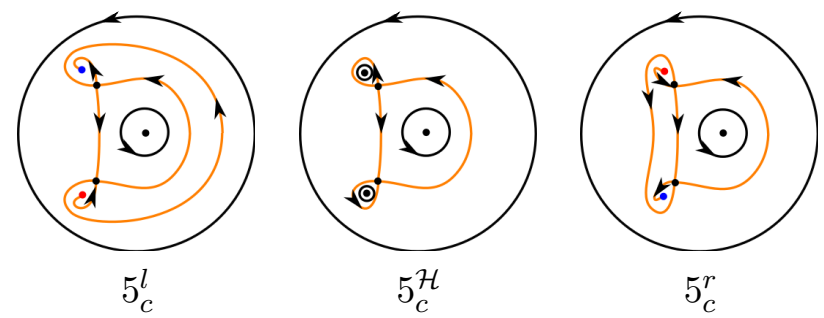

FiguRE 21. Phase portraits in $\mathcal{P}_{+} \cap \mathcal{G}_{r} \cap \mathcal{E}_{u} \cap \mathcal{F}_{d}$ (referred to as $5_{c}$ ).

Proof. The proofs of cases $5_{u}, 5_{c}$ and $5_{d}$ are similar to the proof of case $7_{d}$ in Proposition 20. First we use arguments of continuity to prove the existence of the corresponding global phase portraits. Next, using the symmetry, we study the possible intersections of the separatrices at the saddle along $\mathcal{R}_{0}$ and the saddle-node along $\mathcal{R}_{+}$(resp. at the symmetric saddles along $\mathcal{R}_{+}$and $\mathcal{R}_{-}$) in cases $5_{u}$ and $5_{d}$ (resp. $5_{c}$ ) in the upper plane $y>0$, using pictures similar to Figures 18 and 19 . 

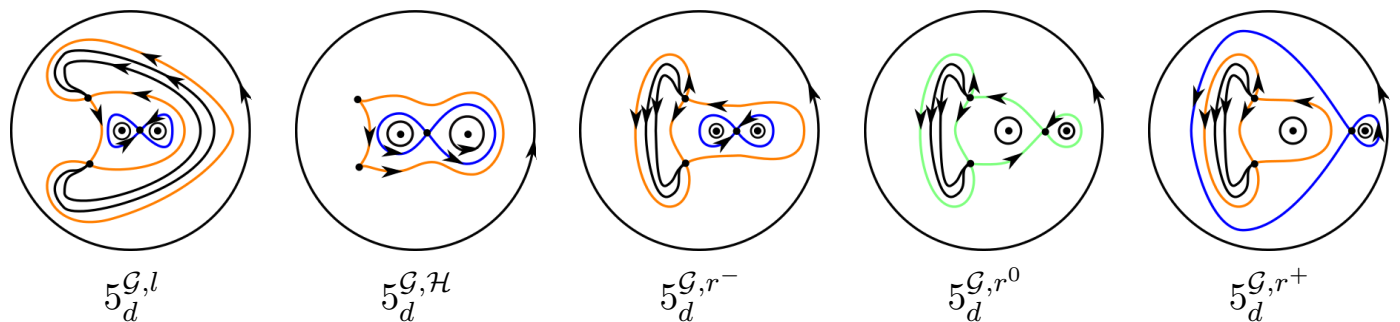

Figure 22. Phase portraits in $\mathcal{P}_{+} \cap \mathcal{G} \cap \mathcal{E}_{d}$ (referred to as $5_{d}$ ).

First we deal with case $5_{u}$. Bifurcating from $7_{u}^{l^{*}}$ (resp. $7_{u}^{l^{-}}$) to $3_{u}$ there exists $5_{u}^{\mathcal{G}, l^{+}}$ (resp. $5_{u}^{\mathcal{G}, l^{-}}$). Next by continuity from $5_{u}^{\mathcal{G}, l^{+}}$to $5_{u}^{\mathcal{G}, l^{-}}$also $5_{u}^{\mathcal{G}, l^{0}}$ exists. Based on the relative positions of the separatrices at the saddle-node and the saddle, the attracting nature of the nodal part of $\mathbf{s}^{\mathbf{1}}$, Proposition 15 and the reversibility property, it turns out that these are the only possible phase portraits. Now by comparing these phase portraits in $\mathcal{G} \cap \mathcal{F}_{u}$, it is seen that, up to topological equivalence, there are exactly 3 that are shortly referred to as $5_{u}$.

Next we deal with case $5_{c}$. Bifurcating from the Hamiltonian case $5_{c}^{H}$ there exist $5_{c}^{l}$ and $5_{c}^{r}$. That these are the only possible phase portraits follows as in case $5_{u}$, considering now the relative positions of the separatrices at the saddle and the node and the attracting nature of $\mathbf{s}^{\mathbf{1}}$. Now by comparing these phase portraits in $\mathcal{G}_{r} \cap \mathcal{E}_{u} \cap \mathcal{F}_{d}$, it is seen that, up to topological equivalence, there are exactly 2 that are shortly referred to as $5_{c}$.

Next we deal with case $5_{d}$. Bifurcating from $7_{d}^{l^{-}}$(resp. $7_{d}^{r^{-}}$and $7_{d}^{r^{+}}$) and $3_{d}$, there exists $5_{d}^{\mathcal{G}, l}$ (resp. $5_{d}^{\mathcal{G}, r^{-}}$and $5_{d}^{\mathcal{G}, r^{+}}$). Next by continuity from $5_{d}^{\mathcal{G}, r^{-}}$to $5_{d}^{\mathcal{G}, r^{+}}$also $5_{d}^{\mathcal{G}, r^{0}}$ exists.

Finally from the study of the separatrices at the saddle $\mathbf{s}_{-}^{0}$ and the saddle-node $\mathbf{s}^{1}$ in $\mathcal{G} \cap \mathcal{E}_{d}$ there are exactly 4 in $\mathcal{G} \cap \mathcal{E}_{d} \cap\left(\mathcal{H}_{r} \cup \mathcal{H}\right)$ and there are 5 possibilities in $\mathcal{G} \cap \mathcal{E}_{d} \cap \mathcal{H}_{l}$ for the intersection of $\mathcal{U}_{E}\left(\mathbf{s}_{-}^{0}\right)$ with $\mathcal{R}_{+}$in forward time, each giving rise to exactly 1 global phase portrait; this can be seen by a picture similar to Figure 18(c). All these 5 phase portraits are topologically mutually distinct. It is seen that 1 of these phase portraits is $5_{d}^{\mathcal{G}, l}$, and thus is equivalent to $5_{d}^{\mathcal{G}, r^{+}}$. Another is equivalent to $5_{d}^{\mathcal{G}, r^{-}}$, and the other 3 are different from any of the phase portraits in Figures 18, 19 and 20. Therefore there are $4 \leq n \leq 7$ in $\mathcal{G} \cap \mathcal{E}_{d}$ and $9 \leq n \leq 12$ with five singularities.

However, as can be seen in the bifurcation diagram drawn in Figure 33 in Section 7.6, the region $\mathcal{G} \cap \mathcal{E}_{d} \cap \mathcal{H}_{l}$ is far from the region $7_{d}^{l^{+}}$and therefore it is believed that the lower bounds in Proposition 21 are the exact numbers.

\subsection{Six singularities.}

Proposition 22. Let $\zeta \in \mathcal{P}_{+}$. The phase portrait of (2) presents six singularities for $\zeta \in(\mathcal{E} \cup \mathcal{F}) \cap \mathcal{G}_{r}$. In this case there are at least $8 \leq n \leq 9$ topologically different phase portraits.

(1) There are exactly 4 in $\mathcal{F} \cap \mathcal{G}_{r}$ as shown in Figure 24 and are referred to as $6_{u}$. In particular, $6_{u}^{l}$ and $6_{u}^{r^{-}}$are equivalent.

(2) There are $6 \leq n \leq 7$ in $\mathcal{E} \cap \mathcal{G}_{r}$. More precisely, there are exactly 6 in $\mathcal{E} \cap \mathcal{G}_{r} \cap$ $\left(\mathcal{H}_{l} \cup \mathcal{H}\right)$ and $1 \leq n \leq 3$ in $\mathcal{E} \cap \mathcal{G}_{r} \cap \mathcal{H}_{r}$. These phase portraits are shown in Figure 25 and are referred to as $6_{d}$. In particular, $6_{d}^{r}$ and $6_{d}^{l^{+}}$are equivalent. In particular, $6_{u}^{l}$ and $6_{d}^{r}$ are equivalent, as also $6_{u}^{\mathcal{H}}$ and $6_{d}^{\mathcal{H}}$ are. 


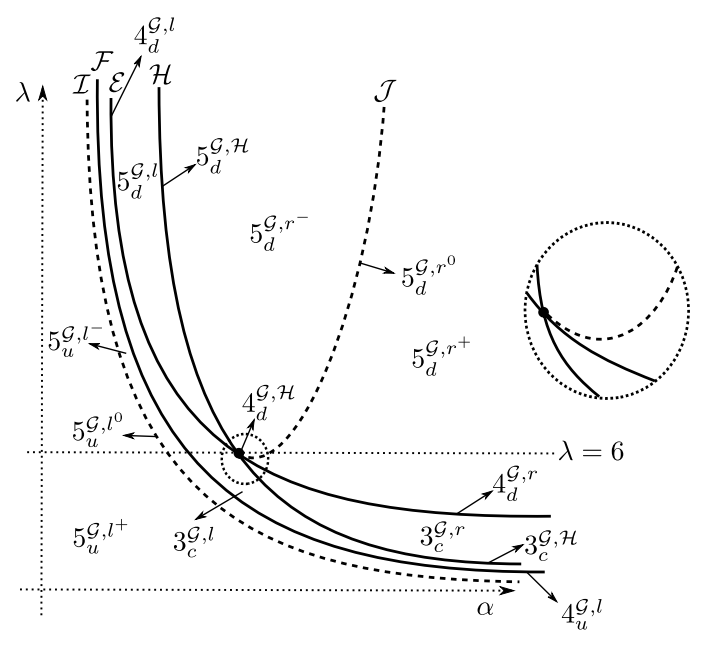

FiguRE 23. Bifurcation diagram restricted to $\mathcal{G}$ projected in the $(\alpha, \lambda)$ plane, i.e. $\pi_{2}\left(\mathcal{G} \cap \mathcal{P}_{+}\right)$.
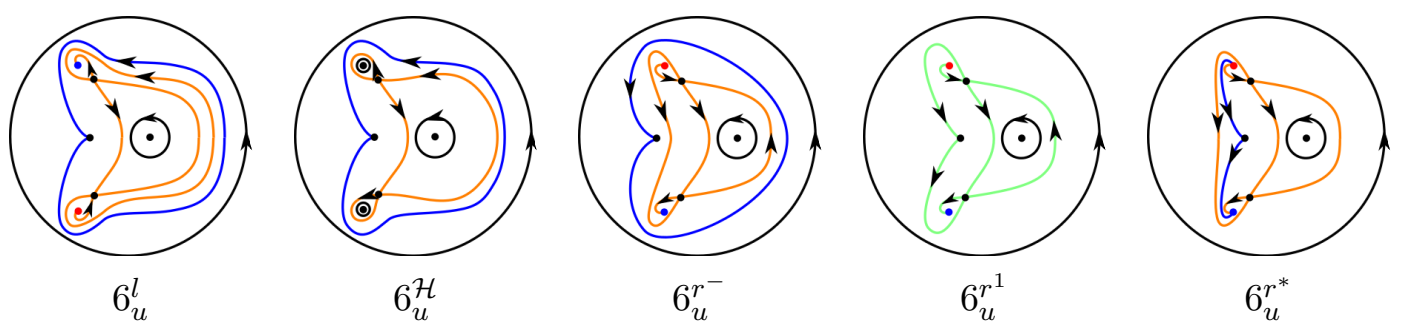

FiguRE 24. Phase portraits in $\mathcal{P}_{+} \cap \mathcal{G}_{r} \cap \mathcal{F}$ (referred to as $6_{u}$ ).
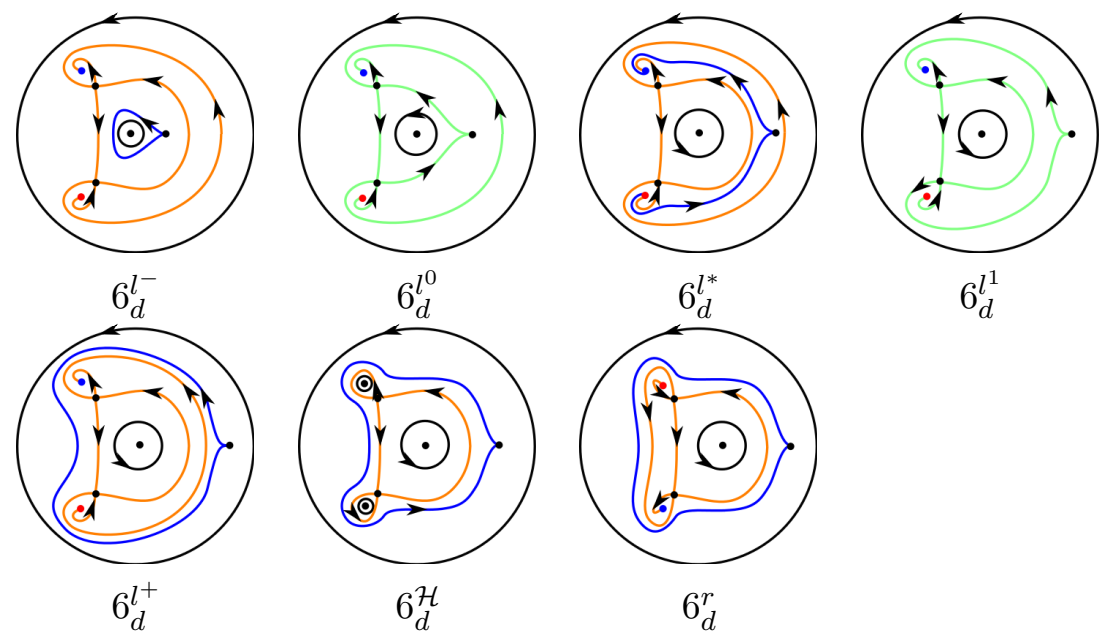

FiguRE 25. Phase portraits in $\mathcal{P}_{+} \cap \mathcal{G}_{r} \cap \mathcal{E}$ (referred to as $6_{d}$ ).

Proof. The proofs of cases $6_{u}$ and $6_{d}$ are similar to the proof of case $7_{d}$ in Proposition 20. First we use arguments of continuity to prove the existence of the corresponding global phase portraits. Next, using the symmetry, we study the possible intersections of the 
separatrices at the cusp $\mathbf{s}^{0}$ and the saddle $\mathbf{s}_{-}^{1}$ in the upper plane $y>0$, using pictures similar to the ones in Figures 18 and 19.

First we deal with case $6_{u}$. Bifurcating from the Hamiltonian case $6_{u}^{\mathcal{H}}$ there exist $6_{u}^{l}$ and $6_{u}^{r^{-}}$. Next by continuity from $5_{c}^{r}$ and $7_{u}^{r^{*}}$ also $6_{u}^{r^{*}}$ exists. As a consequence, by continuity from $6_{u}^{r^{-}}$and $6_{u}^{r^{*}}$ there exists $6_{u}^{r^{1}}$.

Next we deal with case $6_{d}$. Bifurcating from the Hamiltonian case $6_{d}^{\mathcal{H}}$, there exist $6_{d}^{l^{+}}$and $6_{d}^{r}$. Next by continuity from $5_{c}^{l}$ and $7_{d}^{l^{-}}$also $6_{d}^{l^{-}}$exists. As a consequence, by continuity from $6_{d}^{l^{-}}$and $6_{d}^{l^{+}}$, there exist $6_{d}^{l^{0}}, 6_{d}^{l^{*}}$ and $6_{d}^{l^{1}}$.

Based on the relative positions of the separatrices at the cusp and the saddle, the attracting resp. repelling nature of $\mathbf{s}_{+}^{1}$, Proposition 15 , the reversibility property and continuity, it turns out that $6_{u}^{l}, 6_{u}^{\mathcal{H}}, 6_{u}^{r^{-}}, 6_{u}^{r^{0}}$ and $6_{u}^{r^{+}}$are the only phase portraits in $\mathcal{F} \cap \mathcal{G}_{r}$. Analogously, it turns out that $6_{d}^{l^{-}}, 6_{d}^{l^{0}}, 6_{d}^{l^{*}}, 6_{d}^{l^{1}}, 6_{d}^{l^{+}}, 6_{d}^{\mathcal{H}}$, and $6_{d}^{r}$ are the only phase portraits in $\mathcal{E} \cap \mathcal{G}_{r} \cap\left(\mathcal{H}_{l} \cup \mathcal{H}\right)$.

Finally, analyzing the relative positions of the separatrices at the saddle and cusp as above there are only 2 more phase portraits possible in $\mathcal{E} \cap \mathcal{G}_{r} \cap \mathcal{H}_{r}$ than $6_{d}^{r}$, the one that is drawn in Figure 25. These other possibilities are determined by the relative position of the intersection of the unstable separatrix at the cusp with the ray $\mathcal{R}_{+}$. Firstly, this intersection point is at distance smaller than $r_{-}^{1}$ from the origin, giving rise to $6_{d}^{l^{-}}$topologically; secondly, the intersection point is at distance larger than $r_{-}^{1}$ from the origin, giving rise to a phase portrait that is topologically different from $6_{d}^{r}$ and $6_{d}^{l^{-}}$and all other phase portraits with six singularities. Therefore there are at most 3 topologically different phase portraits in $\mathcal{E} \cap \mathcal{G}_{r} \cap \mathcal{H}_{r}$. Furthermore, since $6_{d}^{r}$ is topologically equivalent to $6_{d}^{l^{+}}$, there are at least 6 and at most 7 phase portraits in $\mathcal{E} \cap \mathcal{G}_{r}$. Comparing the phase portraits of $6_{u}$ and $6_{d}$ we find furthermore that topologically $6_{u}^{l}=6_{d}^{l^{+}}, 6_{u}^{\mathcal{H}}=6_{d}^{\mathcal{H}}$ and $6_{u}^{r^{-}}=6_{d}^{r}$; hence there are at least 8 and at most 9 phase portraits with six singularities.

In Figures 26 and 27 the bifurcation diagram is drawn restricted to $\mathcal{E}$ and $\mathcal{F}$ in the $(\alpha, \lambda)$-plane respectively. In particular the bifurcation surfaces $\mathcal{G}, \mathcal{H}, \mathcal{J}, \mathcal{K}$ and $\mathcal{L}$, defined in (4), (29) and (30), also are drawn restricted to $\mathcal{E}$ and $\mathcal{F}$; the dashed lines are used to indicate those bifurcation curves that are numerically obtained.

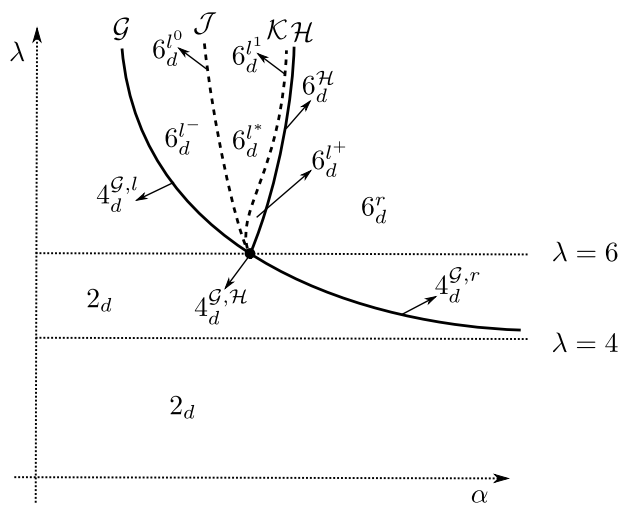

FiguRE 26. Bifurcation diagram restricted to $\mathcal{E}$ projected in the $(\alpha, \lambda)$ plane, i.e. $\pi_{2}\left(\mathcal{E} \cap \mathcal{P}_{+}\right)$. 


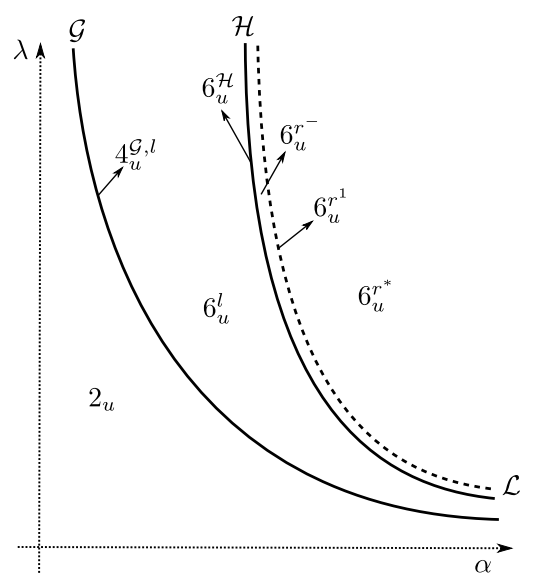

FiguRE 27. Bifurcation diagram restricted to $\mathcal{F}$ projected in the $(\alpha, \lambda)$ plane, i.e. $\pi_{2}\left(\mathcal{F} \cap \mathcal{P}_{+}\right)$.

From this proof it is seen that there cannot exist more bifurcation curves in the region of six singularities neither in Figure 27 neither in the region $\mathcal{G}_{r} \cap \mathcal{H}_{l} \cap \mathcal{E}$ in Figure 26, that is referred to as $6_{d}^{l}$. However from this proof one cannot conclude whether there are more bifurcation curves in the region $\mathcal{G}_{r} \cap \mathcal{H}_{r} \cap \mathcal{E}$ in Figure 26, that is referred to as $6_{d}^{r}$.

Fixing concrete values of $\lambda$, increasing $\alpha$ and studying the relative positions of the separatrices at the cusp and the saddle, we find that the hyperbolic separatrix $\mathcal{W}_{E}\left(\mathbf{s}_{-}^{1}\right)$ intersects the ray $\mathcal{R}_{0}$ on the left of the cusp. Therefore numerically no other bifurcations are found. Taking into account the numerically obtained bifurcation diagram in Figure 28 they cannot appear since $5_{c}^{r}$ and $7_{d}^{r^{+}}$are the only that are nearby.

7.5. Four singularities. When $Y_{\zeta}$ has exactly four singularities the local phase portraits can be $4_{d}^{\mathcal{G}, \mathcal{H}}, 4_{d}^{\mathcal{G}, r}, 4_{d}^{\mathcal{G}, l}$ or $4_{u}^{\mathcal{G}, l}$. From Theorem 17 we already know that the local phase portrait $4_{d}^{\mathcal{G}, \mathcal{H}}$ uniquely determines the global phase portrait and it is drawn in Figure 28. Here we obtain the uniqueness of the global phase portrait in case of the local phase portrait $4_{u}^{\mathcal{G}, l}$, that is proven in Proposition 23 below. In case of the local phase portraits $4_{d}^{\mathcal{G}, r}$ and $4_{d}^{\mathcal{G}, l}$ we have strong numerical evidence that the global phase portraits are unique as well in these cases and are as drawn in Figure 28; this is explained after proving Proposition 23.

Notice that the vector fields having exactly four singularities correspond to $\mathcal{G} \cap(\mathcal{E} \cup \mathcal{F})$ and determine the 1-parameter family $Y_{(\alpha, \gamma(\alpha), \lambda(\alpha))}$, where

$$
\gamma(\alpha)=2 \sqrt{\alpha^{2}+1} / \alpha \text { and }(\lambda(\alpha)=\gamma(\alpha)+2 \text { or } \lambda(\alpha)=\gamma(\alpha)-2) .
$$

Proposition 23. Let $\zeta \in \mathcal{P}_{+}$. The phase portrait of (2) has four singularities if and only if $\zeta \in \mathcal{G} \cap(\mathcal{E} \cup \mathcal{F})$. In this case there are $3 \leq n \leq 6$ topologically different phase portraits that are drawn in Figure 28.

(1) There exists exactly 1 in $\mathcal{G} \cap \mathcal{F}$ and 1 in $\mathcal{G} \cap \mathcal{H}$. These are denoted respectively as $4_{u}^{\mathcal{G}, l}$ and $4_{d}^{\mathcal{G}, \mathcal{H}}$.

(2) There exist $2 \leq n \leq 5$ in $(\mathcal{G} \cap \mathcal{E}) \backslash \mathcal{H}$. In particular, there are $1 \leq n \leq 3$ in $\mathcal{G} \cap \mathcal{E} \cap \mathcal{H}_{r}$ of which $4_{d}^{\mathcal{G}, r}$ is one and there are $1 \leq m \leq 5$ in $\mathcal{G} \cap \mathcal{E} \cap \mathcal{H}_{l}$ of which 
$4_{d}^{\mathcal{G}, l}$ is one. Furthermore all possible phase portraits in $\mathcal{G} \cap \mathcal{E} \cap \mathcal{H}_{r}$ are possible ones in $\mathcal{G} \cap \mathcal{E} \cap \mathcal{H}_{l}$.

In particular, $4_{d}^{\mathcal{G}, r}$ and $4_{u}^{\mathcal{G}, l}$ are equivalent.
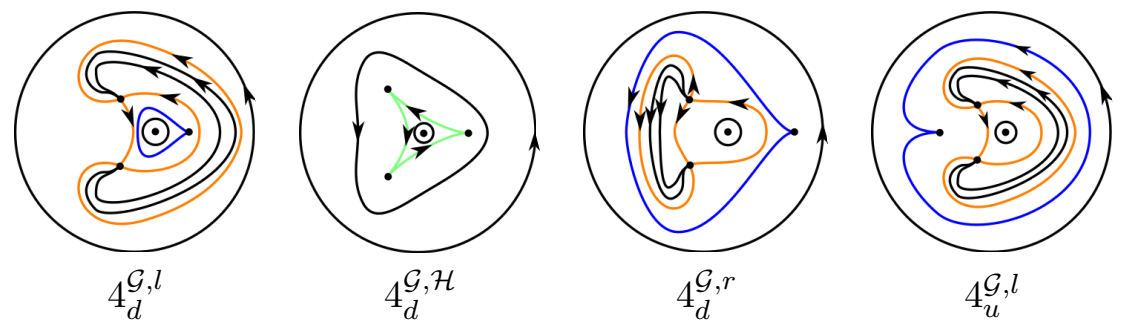

Figure 28. Phase portraits in $\mathcal{G} \cap(\mathcal{E} \cup \mathcal{H} \cup \mathcal{F})$.

Proof. The Hamiltonian case has already been treated in Section 5, so we are left with the existence of the phase portraits $4_{u}^{\mathcal{G}, l}, 4_{d}^{\mathcal{G}, r}$ and $4_{d}^{\mathcal{G}, l}$ and the unicity of the first one. The existence part follows reasoning in the same way as in the proof of Proposition 22 using a continuity argument from the existence of corresponding phase portraits with three and five singularities. Therefore we concentrate on the unicity of $4_{u}^{\mathcal{G}, l}$ and to end we briefly describe the different possibilities in $\mathcal{G} \cap \mathcal{E}$ to give a fine upper bound for the total number of phase portraits with four singularities.

Let $\zeta$ such that the local phase portrait near $\mathcal{R}_{0}$ and $\mathcal{R}_{+}$is given by $4_{u}^{\mathcal{G}, l}$. This corresponds to

$$
\gamma=\frac{2 \sqrt{\alpha^{2}+1}}{\alpha} \text { and } \lambda=\frac{2 \sqrt{\alpha^{2}+1}}{\alpha}-2 .
$$

The unique singularity along both $\mathcal{R}_{ \pm}$is a saddle-node corresponding to $\mathcal{G} \cap \mathcal{H}_{l}$ and the unique singularity along $\mathcal{R}_{0}$ is a cusp corresponding to $\mathcal{F}$. Consider the straight line $L$ through the singularities $\mathbf{s}^{0}$ and $\mathbf{s}^{1}$. Let $\Delta\left(\mathbf{0}, \mathbf{s}^{0}, \mathbf{s}^{1}\right)$ be the triangle bounded by $\mathcal{R}_{0}, L$ and $\mathcal{R}_{+}$as illustrated in Figure 29(a).

The unstable separatrix $\mathcal{U}_{I}\left(\mathbf{s}^{1}\right)$ is tangent to $\mathcal{R}_{+}$and lies inside the triangle $\Delta\left(\mathbf{0}, \mathbf{s}^{0}, \mathbf{s}^{1}\right)$ for reversed time sufficiently large $(t \rightarrow-\infty)$. The stable separatrix $\mathcal{W}_{E}\left(\mathbf{s}^{0}\right)$ is tangent to $\mathcal{R}_{0}$ and lies in $E$ but outside the triangle $\Delta\left(\mathbf{0}, \mathbf{s}^{0}, \mathbf{s}^{1}\right)$ for times sufficiently large $(t \rightarrow \infty)$. The orbit $\mathcal{U}_{I}\left(\mathbf{s}^{1}\right)$ will leave the triangle $\Delta\left(\mathbf{0}, \mathbf{s}^{0}, \mathbf{s}^{1}\right)$ only by crossing $\mathcal{R}_{0}$ between $\mathbf{0}$ and $\mathbf{s}_{0}$. Indeed suppose to the contrary that $\mathcal{U}_{I}\left(\mathbf{s}^{1}\right)$ would leave the triangle by first crossing $L$ between $\mathbf{s}^{0}$ and $\mathbf{s}^{1}$. This would imply the existence of four tangency points along $L$ that are two by two distinct (including the singularities $\mathbf{s}^{0}$ and $\mathbf{s}^{1}$ ), see Figure 29(b). This is in contradiction with the fact that the system is cubic and irreducible and therefore can have at most three tangency points, see e.g. [10]. Hence by symmetry the orbit $\mathcal{U}_{I}\left(\mathbf{s}^{1}\right)$ connects the saddle-nodes $\mathbf{s}^{1}$ and $\mathbf{s}^{2}$ inside the polygon $\mathbf{0}, \mathbf{s}^{1}, \mathbf{s}^{0}, \mathbf{s}^{2}$. Now the global phase portrait $4_{u}^{\mathcal{G}, l}$ as presented in Figure 28 clearly is uniquely determined.

Now we count the phase portraits with four singularities. There is exactly 1 in $\mathcal{G} \cap \mathcal{F}$ and 1 in $\mathcal{G} \cap \mathcal{H}$ referred to as $4_{u}^{\mathcal{G}, l}$ and $4_{d}^{\mathcal{G}, \mathcal{H}}$ respectively. There are 3 (resp. 5) possible phase portraits in $\mathcal{G} \cap \mathcal{E} \cap \mathcal{H}_{r}$ (resp. $\mathcal{G} \cap \mathcal{E} \cap \mathcal{H}_{l}$ ), and the 3 possible ones in $\mathcal{G} \cap \mathcal{E} \cap \mathcal{H}_{r}$ are topologically equivalent to 3 of the 5 possible ones in $\mathcal{G} \cap \mathcal{E} \cap \mathcal{H}_{l}$. One of the phase portraits in $\mathcal{G} \cap \mathcal{E} \cap \mathcal{H}_{r}, 4_{d}^{\mathcal{G}, r}$, is equivalent to $4_{u}^{\mathcal{G}, l}$. Therefore there are at least 3 and at most 6 phase portraits with four singularities. 


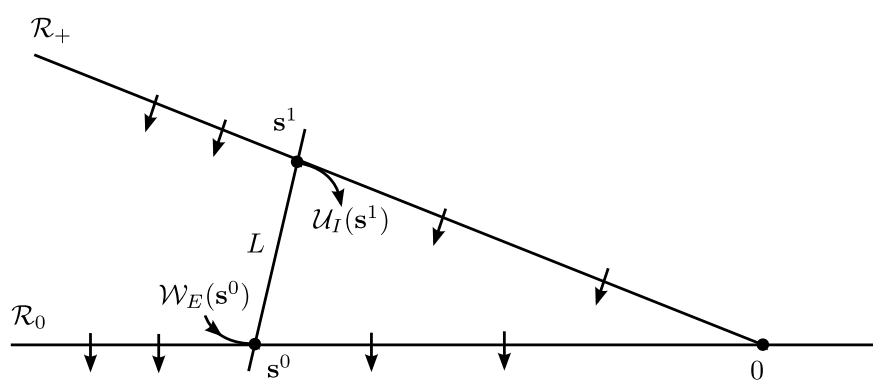

(a)

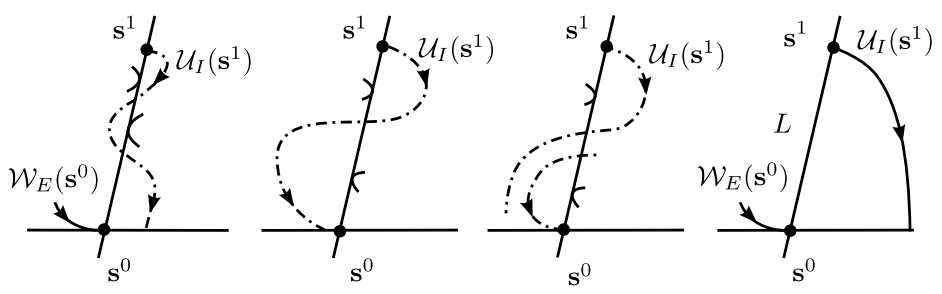

Figure 29. (a) Triangle $\Delta\left(\mathbf{0}, \mathbf{s}^{0}, \mathbf{s}^{1}\right)$ in the phase plane; (b) Four tangency points along the straight line $L$ if $\mathcal{U}_{I}\left(\mathbf{s}^{1}\right)$ cuts $L$ in forward time.

The crucial part in proving the global phase portrait $4_{u}^{\mathcal{G}, l}$ is to guarantee the relative position of the separatrices $\mathcal{U}_{I}\left(\mathbf{s}^{1}\right)$ and $\mathcal{W}_{E}\left(\mathbf{s}^{0}\right)$. We showed analytically that there is only one possibility for it. In Figure 30 the forward and backward intersections of respectively $\mathcal{U}_{I}\left(\mathbf{s}^{1}\right)$ and $\mathcal{W}_{E}\left(\mathbf{s}^{0}\right)$ with the rays $\mathcal{R}_{0}$ and $\mathcal{R}_{+}$are drawn for the 1 -parameter family $Y_{\zeta}, \zeta \in \mathcal{P}_{+} \cap \mathcal{G} \cap \mathcal{F}$, as functions of the parameter $\alpha$, that are numerically obtained using Taylor methods of higher order with sufficiently accuracy. More precisely if the intersection of $\mathcal{U}_{I}\left(\mathbf{s}^{1}\right)$ with ray $\mathcal{R}_{0}$ in forward time happens at distance $\left|u_{\alpha}\right|$ from the origin, the function $u_{\alpha}$ is numerically obtained and its graph is drawn in Figure 30(a). As we prove analytically we find also that $-1<u_{\alpha}<0$ for all $\alpha>0$, it is to say that the separatrix $\mathcal{U}_{I}\left(\mathbf{s}^{1}\right)$ intersects $\mathcal{R}_{0}$ on the right of $\mathbf{s}^{0}$; furthermore numerically we find that the graph is monotone with $\alpha$. Next if the intersection of $\mathcal{W}_{E}\left(\mathbf{s}^{0}\right)$ with ray $\mathcal{R}_{+}$in backward time happens at distance $w_{\alpha}$ from the origin, the function $w_{\alpha}$ is numerically obtained and its graph is drawn in Figure 30(b). Corresponding to our analytical findings we obtain numerically that $w_{\alpha}>1$ for all $\alpha>0$, it is to say that the separatrix $\mathcal{W}_{E}\left(\mathbf{s}^{0}\right)$ intersects $\mathcal{R}_{+}$in backward time at a distance from the origin bigger than $r^{1}=\left\|\mathbf{s}^{1}\right\|$; furthermore numerically we find that the graph is monotone with $\alpha$.

For $\zeta \in \mathcal{P}_{+} \cap \mathcal{G} \cap \mathcal{E}$ the family $Y_{\zeta}$ is 1-parametric and can be parameterized by $\alpha$ as in (31). Again the key in establishing the global phase portrait is the relative position between the separatrices $\mathcal{W}_{E}\left(\mathbf{s}^{1}\right)$ and $\mathcal{U}_{E}\left(\mathbf{s}^{0}\right)$. We study this numerically using Taylor methods of higher order with sufficiently accuracy. Let $\Sigma$ denote the bisection line between the rays $\mathcal{R}_{0}$ and $\mathcal{R}_{+}$; hence $\Sigma$ is the ray determined by the angle $(\pi-$ $\arctan (1 / \alpha)) / 2$. Let $w_{\alpha}$ denote the first intersection of the separatrix $\mathcal{W}_{E}\left(\mathbf{s}^{1}\right)$ with $\Sigma$ in backward time and let $u_{\alpha}$ denote the first intersection of the separatrix $\mathcal{U}_{E}\left(\mathbf{s}^{0}\right)$ with $\Sigma$ in forward time. Then $\Delta(\alpha)=\left\|u_{\alpha}\right\|-\left\|w_{\alpha}\right\|$ measures the distance between the separatrices $\mathcal{W}_{E}\left(\mathbf{s}^{1}\right)$ and $\mathcal{U}_{E}\left(\mathbf{s}^{0}\right)$ along $\Sigma$ and is found numerically by Taylor methods of sufficiently high accuracy. The graph of the numerically obtained function $\Delta$ is pictured in Figure 31. In particular for $\alpha=1 / \sqrt{3}$ we find numerically that this distance 


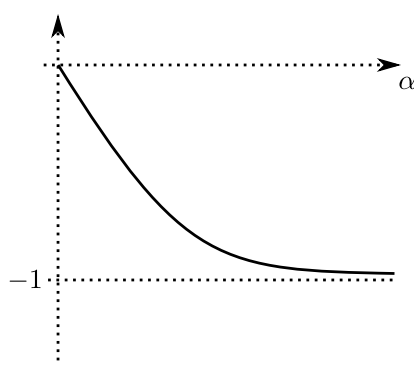

(a)

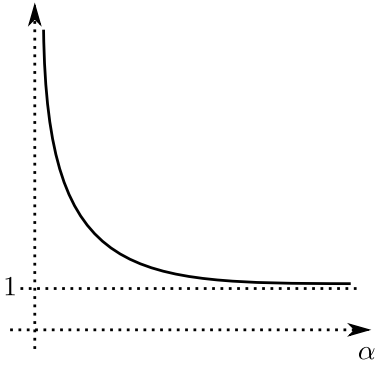

(b)

FiguRE 30. Relative positions of the separatrices $\mathcal{U}_{I}\left(\mathbf{s}^{1}\right)$ and $\mathcal{W}_{E}\left(\mathbf{s}^{0}\right)$ in case $4_{u}^{\mathcal{G}, l}$ determined by $u_{\alpha}$ and $w_{\alpha}$ and whose graphs are drawn respectively in (a) and (b).

is 0 and so that there is a connection between the cusps $\mathbf{s}^{0}$ and $\mathbf{s}^{1}$, as we also found analytically for the Hamiltonian case $4_{d}^{\mathcal{G}, \mathcal{H}}$. For $\alpha \neq 1 / \sqrt{3}$ this graph depicts the relative position of the separatrices $\mathcal{W}_{E}\left(\mathbf{s}^{1}\right)$ at the saddle-node $\mathbf{s}^{1}$ and $\mathcal{U}_{E}\left(\mathbf{s}^{0}\right)$ at the cusp $\mathbf{s}^{0}$. As a consequence in case $\alpha<1 / \sqrt{3}$ the separatrix $\mathcal{W}_{E}\left(\mathbf{s}^{1}\right)$ intersects the ray $\mathcal{R}_{0}$ in backward time on the right of $\mathbf{s}^{0}$, while for $\alpha>1 / \sqrt{3}$ it does so on the left of $\mathbf{s}^{0}$. Using the symmetry of $Y_{\zeta}$ the global phase portraits are uniquely determined by the local ones $4_{d}^{\mathcal{G}, l}$ and $4_{d}^{\mathcal{G}, r}$ for $\alpha<1 / \sqrt{3}$ and $\alpha>1 / \sqrt{3}$ respectively as drawn in Figure 28.

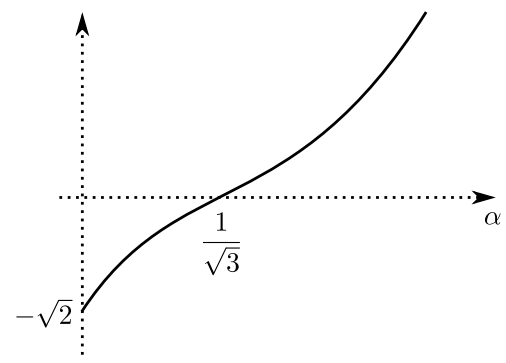

FiguRE 31. Graph of the distance between the intersection of the separatrices $\mathcal{W}_{E}\left(\mathbf{s}^{1}\right)$ and $\mathcal{U}_{E}\left(\mathbf{s}^{0}\right)$ with the line of slope $(\pi-\arctan (1 / \alpha)) / 2$ in case $4_{d}^{\mathcal{G}}$.

7.6. Global bifurcation diagram in $\mathcal{P}_{+}$. From the previous sections we know that the global bifurcation diagram is obtained from the local one adding the bifurcation surfaces $\mathcal{I}, \mathcal{J}, \mathcal{K}$ and $\mathcal{L}$. Here we concentrate on slices of the global bifurcation diagram for fixed $\lambda>0$ in the $(\alpha, \gamma)$-plane. As seen in Figure 7, the slices of the local bifurcation diagram for fixed $\lambda$ do not change qualitatively in the region $\gamma>\gamma^{*}(\lambda)$, where

$$
\gamma^{*}(\lambda)=\frac{\lambda+2+\max \left\{\gamma_{0}, \lambda-2\right\}}{2}
$$

and $\gamma_{0}(\lambda)$ the unique positive solution of $\gamma^{3}-4 \gamma-8 \lambda=0$. It does so for $\gamma<\gamma^{*}(\lambda)$ and adding the global bifurcation curves there are 7 typical slices to be distinguished. Then the slices of the global bifurcation diagram for fixed $\lambda$ is presented over Figures 32 and 33 for $\left\{\alpha>0, \gamma \geq \gamma^{*}(\lambda)\right\}$ and $\left\{\alpha>0,0<\gamma \leq \gamma^{*}(\lambda)\right\}$ respectively. In both figures analytically obtained curves are drawn by continuous lines and numerically obtained ones 
by dashed lines. The different bifurcation regions are identified by the corresponding global phase portraits as they are referred to in Propositions 19, 20, 21, 22 and 23 and the corresponding figures. Notice furthermore that the phase portraits $n_{u}$ (resp. $n_{d}$ ) are included in $\gamma \geq \gamma^{*}(\lambda)$ (resp. $0<\gamma \leq \gamma^{*}(\lambda)$ ).

The typical slice of the global bifurcation diagram for fixed $\lambda>0$ restricted to $\left\{\alpha>0, \gamma \geq \gamma^{*}(\lambda)\right\}$ is obtained from the local one adding the corresponding slices of $\mathcal{I}$ and $\mathcal{L}$, that we found in (30). These curves are obtained numerically for some concrete values of $\lambda$, for which each of these curves show the typical behavior drawn in Figure 32. In particular we find that $\mathcal{L}$ is very close to $\mathcal{H}$, which makes its search more delicate. Both bifurcation surfaces originate from $7_{u}^{\mathcal{H}^{0}}$, both having one branch in $\mathcal{H}_{l}$ and one in $\mathcal{H}_{r}$. The branch of $\mathcal{I}$ in $\mathcal{H}_{l}$ dies in $\mathcal{G}$ and the branch of $\mathcal{L}$ in $\mathcal{H}_{r}$ dies in $\mathcal{F}$.

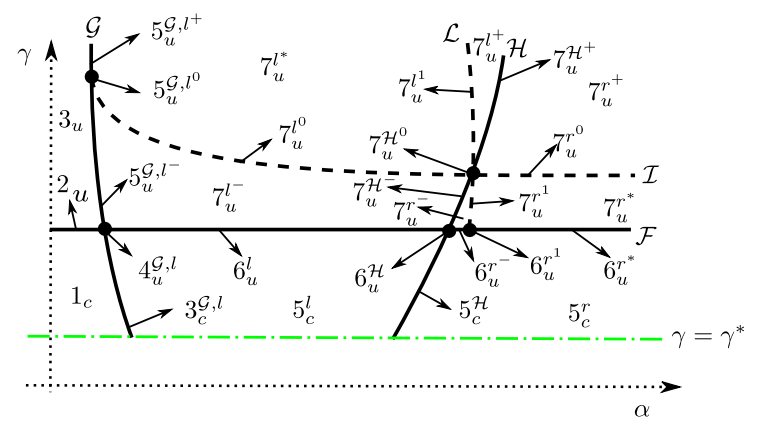

FiguRE 32. Characteristic slice of the global bifurcation diagram for fixed $\lambda>0$ restricted to $\left\{\alpha>0, \gamma \geq \gamma^{*}(\lambda)\right\}$.

Next the slices of the global bifurcation diagram for fixed $\lambda>0$ restricted to $\{\alpha>$ $\left.0,0<\gamma<\gamma^{*}(\lambda)\right\}$ are obtained from the local one adding $\mathcal{J}$ and $\mathcal{K}$ as we found in (29). These curves are obtained numerically for some concrete values of $\lambda$. Depending to which $\lambda$-range it belongs, the curves $\mathcal{J}$ and $\mathcal{K}$ are present and have the typical behavior shown in Figure 33. Furthermore we found that the curves $\mathcal{K}$ and $\mathcal{H}$ are very close and their distance decreases with increasing $\lambda$, as is shown in Figure 26. In fact we can be more precise. From the zoom in Figure 23 it is seen that the slice of $\mathcal{J}$ in $\mathcal{G}$ presents a minimum $\lambda^{*}$ in the $(\alpha, \lambda)$-plane. This $\lambda^{*}$ is approximately equal to 5.98323 and it is taken by $\alpha^{*} \approx 0.59405$. For $0<\lambda<\lambda^{*}$ the bifurcation diagram is determined by the local one. For $\lambda \geq \lambda^{*}$ the bifurcation surfaces $\mathcal{J}$ and $\mathcal{K}$ appear. In Figure 26 it is seen that besides the fact that the curves $\mathcal{H}$ and $\mathcal{K}$ are very close also the distance between them decreases for increasing $\lambda$.

For $\lambda>6$ the bifurcation surfaces originate from $7_{d}^{\mathcal{H}^{0}}$, both having one branch in $\mathcal{H}_{l}$ ending in $\mathcal{E}$ while $\mathcal{J}$ also has a branch in $\mathcal{H}_{r}$ ending in $\mathcal{G}$. In particular, based on Figure 33 , it can be concluded that there is only 1 phase portrait for $5_{d}^{\mathcal{G}, l}$ and for $6_{d}^{r}$ by arguments of continuity as was observed in Section 7.4. Indeed the other possibilities for $5_{d}^{\mathcal{G}, l}$ (resp. $6_{d}^{r}$ ) would appear by continuity from $3_{d}$ to $7_{d}^{l^{+}}$(resp. $5_{c}^{r}$ to $7_{d}^{r^{-}}$), that are separated by an open set having another configuration.

When $\lambda$ decreases, the area of the region $7_{d}^{l}$ (resp. $7_{d}^{r^{-}}$) shrinks and collapses to the point $4_{d}^{\mathcal{G}, \mathcal{H}}$ for $\lambda=6$ (resp. $5_{d}^{\mathcal{G}, r^{0}}$ for $\lambda=\lambda^{*}$ ) to disappear for $\lambda<6$ (resp. $\lambda<\lambda^{*}$ ).

Besides in Figures 32 and 33 these facts are also seen in Figures 23, 26 and 27, where the slices of $\mathcal{I}$ and $\mathcal{J}$ in $\mathcal{G}$, the slices of $\mathcal{J}$ and $\mathcal{K}$ in $\mathcal{E}$, and the slice of $\mathcal{L}$ in $\mathcal{F}$ respectively are shown in the $(\alpha, \lambda)$-plane. Notice that in Figure 26 (resp. 27) the global bifurcation 
diagram is shown when restricting to $\mathcal{E}$ (resp. $\mathcal{F}$ ), that are included in $\left\{0<\gamma \leq \gamma^{*}(\lambda)\right\}$ (resp. $\left\{\gamma \geq \gamma^{*}(\lambda)\right\}$ ). In particular in Figure 26 the global bifurcation diagram is shown in terms of $(\alpha, \lambda)$ when restricting to the plane $\mathcal{E}$. Fixing $\lambda>0$ in Figure 26 we can follow the mutual intersections of the bifurcation surfaces with $\mathcal{E}$ for increasing $\alpha$. This way, for $\lambda>6$, increasing $\alpha$ along $\mathcal{E}$ the bifurcation surfaces $\mathcal{G}, \mathcal{J}, \mathcal{K}$ and $\mathcal{H}$ are subsequently met. For $\lambda=6$, increasing $\alpha$ along $\mathcal{E}$ only the joint intersection of $\mathcal{G}$ and $\mathcal{H}$ is passed by. For $4<\lambda<6$, increasing $\alpha$ along $\mathcal{E}$ only the bifurcation surface $\mathcal{G}$ is met, while for $0<\lambda \leq 4, \alpha>0$ there are no bifurcation surfaces met.

Similarly in Figure 27 the global bifurcation diagram is shown when restricting to the plane $\mathcal{F}$. Fixing $\lambda>0$ we also see the mutual intersections of the bifurcation surfaces with $\mathcal{F}$ for increasing $\alpha$, that are subsequently $\mathcal{G}, \mathcal{H}$ and $\mathcal{L}$.

However in Figure 23 both $\gamma \geq \gamma^{*}(\lambda)$ and $0<\gamma \leq \gamma^{*}(\lambda)$ are present. To distinguish these cases we notice the following. For fixed $\lambda>0$ there exists a unique $\alpha^{*}(\lambda)$ such that $\left(\alpha^{*}(\lambda), \gamma^{*}(\lambda), \lambda\right) \in \mathcal{G}$ and the curve $\left\{\left(\alpha^{*}(\lambda), \lambda\right): \lambda>0\right\}$ lies above $\pi_{2}(\mathcal{F} \cap \mathcal{G})$ but below $\pi_{2}(\mathcal{H} \cap \mathcal{G}) \cup\left(\pi_{2}(\mathcal{E} \cap \mathcal{G})\right.$. Then $\zeta \in \mathcal{G} \cap\left\{\gamma>\gamma^{*}(\lambda)\right\}$ if and only if $\zeta \in \mathcal{G} \cap\left\{0<\alpha<\alpha^{*}(\lambda)\right\}$, and $\zeta \in \mathcal{G} \cap\left\{0<\gamma<\gamma^{*}(\lambda)\right\}$ if and only if $\zeta \in \mathcal{G} \cap\left\{\alpha>\alpha^{*}(\lambda)\right\}$. Fixing $\lambda>0$, increasing $\alpha$ along $\mathcal{G}$, it is seen in Figure 32 the intersection with $\mathcal{I}$ and $\mathcal{F}$ subsequently, and then continuing in Figure 33 the intersections with $\mathcal{E}, \mathcal{H}$ and $\mathcal{J}$ subsequently. The same can be observed in Figure 23.

\section{CONClusions}

In the previous sections, Theorems 4 and 5 are proven that deal with the classification of the phase portraits of $Y_{\zeta}$, given in (2), restricted to $\mathcal{P}_{ \pm}$. Furthermore, by slicing the bifurcation diagram is obtained in both $\mathcal{P}_{ \pm}$. Here, as before, we write $\zeta=(\alpha, \gamma, \lambda)$ and we consider the extensions $\overline{\mathcal{P}}_{ \pm}=\overline{\mathcal{P}}_{ \pm}^{1} \cup \overline{\mathcal{P}}_{ \pm}^{2}, \overline{\mathcal{P}}=\overline{\mathcal{P}}^{1} \cup \overline{\mathcal{P}}^{2}$ and $\overline{\mathcal{P}}_{0}$ in the parameter space $\mathbb{R}^{3}=\overline{\mathcal{P}} \cup \overline{\mathcal{P}}_{0}$, where

$$
\begin{aligned}
& \overline{\mathcal{P}}_{-}^{1}=\left\{\zeta \in \mathbb{R}^{3}: \gamma=0, \lambda<0\right\} \text { and } \overline{\mathcal{P}}_{-}^{2}=\left\{\zeta \in \mathbb{R}^{3}: \gamma \lambda<0\right\}, \\
& \overline{\mathcal{P}}_{+}^{1}=\left\{\zeta \in \mathbb{R}^{3}: \gamma=0, \lambda>0\right\} \text { and } \overline{\mathcal{P}}_{+}^{2}=\left\{\zeta \in \mathbb{R}^{3}: \gamma \lambda>0\right\}, \\
& \overline{\mathcal{P}}^{i}=\overline{\mathcal{P}}_{-}^{i} \cup \overline{\mathcal{P}}_{+}^{i}, i=1,2 \text { and } \overline{\mathcal{P}}_{0}=\left\{\zeta \in \mathbb{R}^{3}: \lambda=0\right\} .
\end{aligned}
$$

In this section we extend the bifurcation diagram of $Y_{\zeta}$ to $\overline{\mathcal{P}}^{1}, \overline{\mathcal{P}}^{2} \cap\{\alpha=0\}, \overline{\mathcal{P}}_{ \pm}$ and $\overline{\mathcal{P}}_{0}$, that are described in Propositions 24, 25, 27, 28 and 29 respectively. As a consequence we obtain Theorem 1 and Corollary 2, that ask for the classification of $Y_{\zeta}$ for $\zeta \in \overline{\mathcal{P}}=\mathbb{R}^{3} \backslash\{\lambda\}$ and $\zeta \in \mathbb{R}^{3}=\overline{\mathcal{P}} \cup \overline{\mathcal{P}}_{0}$ respectively.

Using results from [9] we obtain the classification on the boundaries $\overline{\mathcal{P}}^{1}$ and $\overline{\mathcal{P}}^{2} \cap\{\alpha=$ $0\}$. The first boundary is the natural extension of $\mathcal{P}_{ \pm}$, described in [9] as the case of three rays with aligned singularities, while the second boundary deals with the case of one simple and one double ray. In both cases by the reversibility property the local phase portrait determines the global phase portrait completely. In this way the following two propositions are proven.

Proposition 24. The subfamily of cubic reversible vector fields $Y_{\zeta}$ for $(2)$ with $\zeta \in \overline{\mathcal{P}}^{1}$, has 3 topologically different global phase portraits that are completely determined by the number of singularities. The classification is listed according to it: $\overline{1}, \overline{2}$ for $\lambda<0$ or $2_{d}$ for $\lambda>0$ and $\overline{3}$, with one, two and three singularities respectively; they happen for $|\lambda|<2,|\lambda|=2$ and $|\lambda|>2$ respectively. 

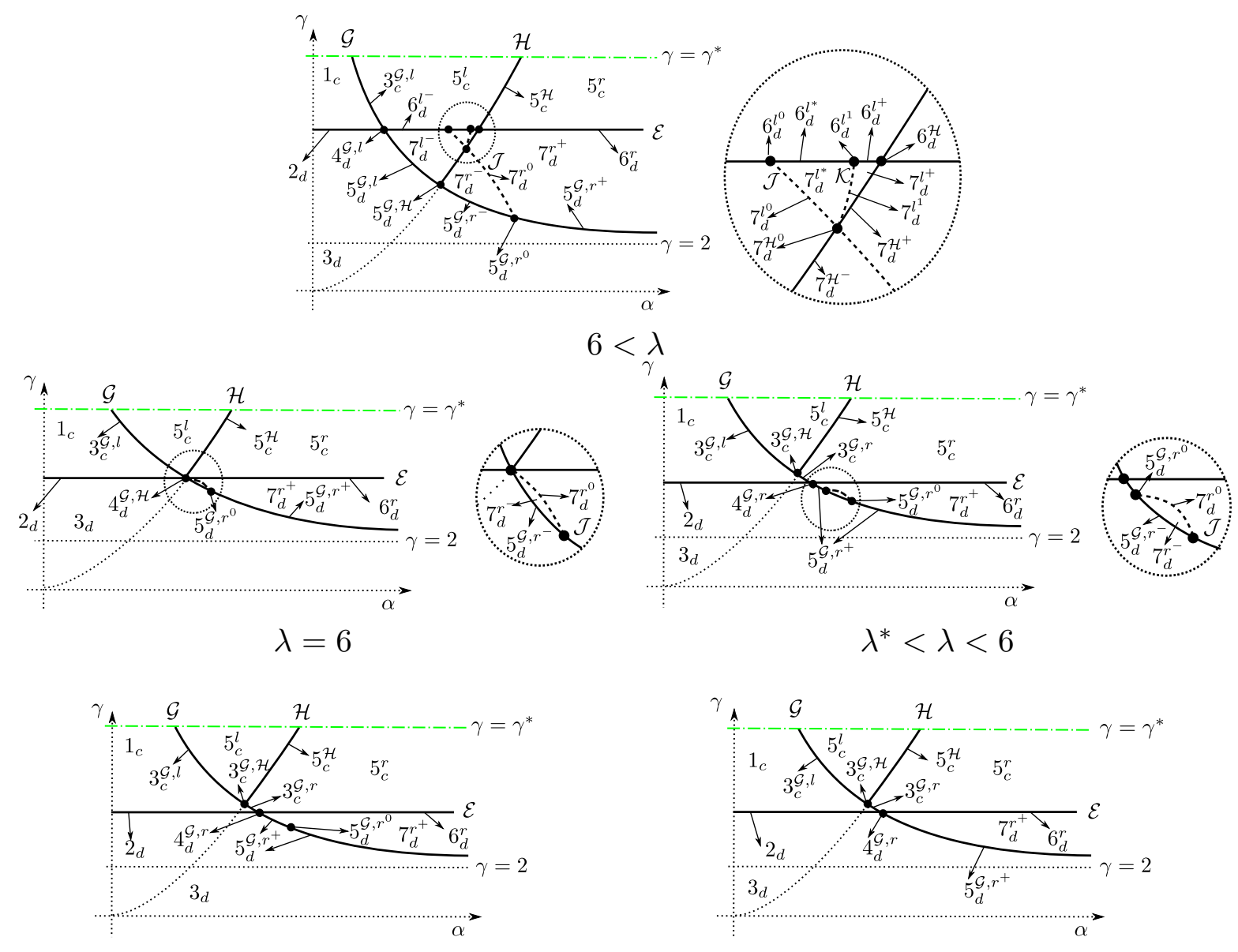

$\lambda=\lambda^{*}$

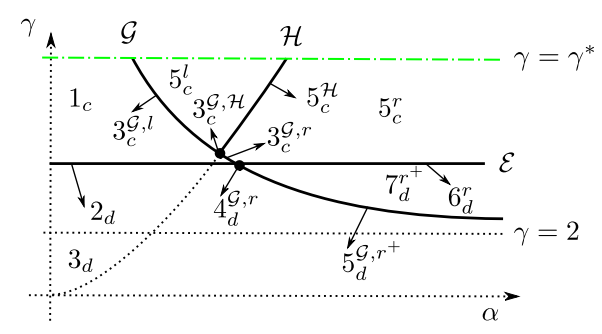

$4<\lambda<\lambda^{*}$

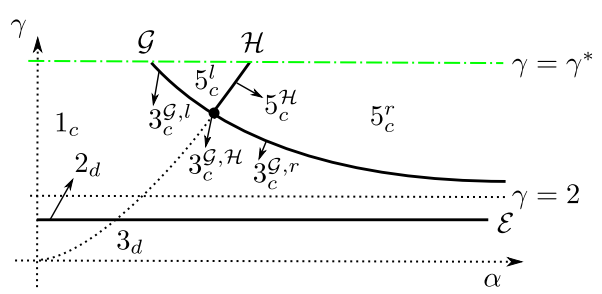

$2<\lambda \leq 4$

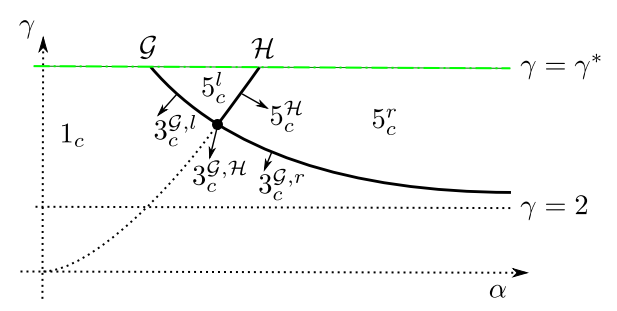

$0<\lambda \leq 2$

FiguRE 33. Characteristic slices of the global bifurcation diagram for fixed $\lambda>0$ restricted to $\left\{\alpha>0,0<\gamma \geq \gamma^{*}(\lambda)\right\}$.

Proposition 25. The subfamily of cubic reversible vector fields $Y_{\zeta}$ for $\zeta \in \overline{\mathcal{P}}^{2} \cap\{\alpha=0\}$, has 4 topologically different global phase portraits. The classification is listed according to the number of singularities as follows: $1_{c}$ for $|\gamma-\lambda|<2 ; \overline{2}$ for $\gamma-\lambda=-2$ or $2_{u}$ for $\gamma-\lambda=2 ; 3_{d}$ for $|\gamma-\lambda|>2$ and $(\gamma-\lambda) \lambda<0$, and $3_{u}$ for $|\gamma-\lambda|>2$ and $(\gamma-\lambda) \lambda>0$ (up to rotation of $180^{\circ}$ for $\gamma-\lambda<0$ ).

The sign of $\alpha$ does not alter the equations, hence by orthogonal reflection about the $(\gamma, \lambda)$-plane and using (11), the bifurcation diagram restricted to $\mathcal{P}_{ \pm}$can be extended to $\overline{\mathcal{P}}^{2} \cap\{\alpha \neq 0\}$. Therefore Proposition 25 gives rise to the following generalization of Theorems 4 and 5 . 
Corollary 26. Theorems 4 and 5 hold true when replacing $\mathcal{P}_{ \pm}$by $\overline{\mathcal{P}}_{ \pm}$respectively.

From Proposition 24 and Corollary 26 we obtain Propositions 27 and 28 establishing the classification in $\overline{\mathcal{P}}_{ \pm}$.

Proposition 27. The subfamily of cubic reversible vector fields $Y_{\zeta}$ for $\zeta \in \overline{\mathcal{P}}_{-}$, has exactly 5 topologically different global phase portraits, that are drawn in Figure 1. Furthermore the global phase portrait of $Y_{\zeta}$ is uniquely determined by the number $n$ of singularities; in particular, up to topological equivalence, the phase portrait is equal to $\bar{n}$ with $n \in\{1,2,3,5,7\}$.

Proposition 28. Theorem 5 evenly holds when replacing $\mathcal{P}_{+}$by $\overline{\mathcal{P}}_{+}$. The subfamily of cubic reversible vector fields $Y_{\zeta}$ for $\zeta \in \overline{\mathcal{P}}_{+}$has at most 53 topologically different global phase portraits of which at least 46 are realized, and that are drawn in Figures 15, 16, 17, 20, 21, 22, 24, 25 and 28.

Passing from $\overline{\mathcal{P}}_{-}$to $\overline{\mathcal{P}}_{+}$we pass through $\overline{\mathcal{P}}_{0}$, and find a bifurcation phenomenon that is already discussed in Section 1 and that is detailed in Proposition 29. Let us therefore recall from [9] that $\infty$ represents, for $\gamma>2$, the phase portrait of a global center at the origin disturbed by a circle of singularities at $(-\gamma / 2,0)$ and radius $\gamma^{2} / 4-1$. Next proposition deals with the classification on $\overline{\mathcal{P}}_{0}$, which has already been dealt with in [9].

Proposition 29. The subfamily of cubic reversible vector fields $Y_{\zeta}$ for $\zeta \in \overline{\mathcal{P}}_{0}$, is 1parametric and has 3 topologically different global phase portraits that are completely determined by the number of singularities. The classification is listed according to it: $1_{c}, 2_{u}$ and $\infty$ for one, two and infinitely many singularities respectively; they happen for $|\gamma|<2,|\gamma|=2$ and $|\gamma|>2$ respectively.

Proof of Theorem 1 and Corollaries 2 and 3. The classification of $Y_{\zeta}$ in $\overline{\mathcal{P}}=\mathbb{R}^{3} \backslash \overline{\mathcal{P}}_{0}$ is obtained by combining Propositions 27 and 28, noticing that $\overline{5}$ and $\overline{7}$ are the only ones in $\overline{\mathcal{P}}_{-}$that do not correspond with any of the ones in $\overline{\mathcal{P}}_{+}$. Then, adding Proposition 29 , Corollary 2 follows. Finally, combining the results from this paper and [8, 9], Corollary 3 follows. Here we take into account that the reversible class in (1) is obtained by adding the triple case studied in [9] to Propositions 27, 28 and 29.

\section{REFERENCES}

[1] A. F. Andreev, Investigation of the behaviour of the integral curves of a system of two differential equations in the neighborhood of a singular point, Translation of Amer. Math. Soc. 8 (1958), $183-207$.

[2] A. A. Andronov, E. A. Leontovich, I. I. Gordon and A. G. Maier, Theory of bifurcations of dynamic systems on a plane, John Wiley \& Sons, New York, 1973.

[3] J. C. Artés, J. Llibre and D. Schlomiuk, The geometry of quadratic differential systems with a weak focus of second order, Internat. J. Bifur. Chaos Appl. Sci. Engrg. 16(11), 3127-3194, 2006.

[4] J. C. Artés, J. Llibre and D. Schlomiuk, The geometry of quadratic differential systems with a weak focus and an invariant straight line, Internat. J. Bifur. Chaos Appl. Sci. Engrg. 20(11), 3627-3662, 2010 .

[5] J. C. Artés, A. C. Rezende and R. D. Oliveira, Global phase portraits of quadratic polynomial differential systems with a semi-elemental triple node, Preprint, 2012.

[6] T. R. Blows and C. Rousseau, Bifurcation at infinity in polynomial vector fields, J. Differential Equations 104, 215-242, 1993.

[7] F. Cao and J. Jiang, The classification on the global phase portraits of two-dimensional LotkaVolterra system, J. Dynam. Differential Equations 20, 797-830, 2008. 
[8] M. Caubergh, J. Llibre and J. Torregrosa, Global classification of a class of cubic vector fields whose canonical regions are period annuli, Internat. J. Bifur. Chaos Appl. Sci. Engrg. 21(7), 1831-1867, 2011.

[9] M. Caubergh, J. Llibre and J. Torregrosa, Global phase portraits of some reversible cubic centers with collinear or infinitely many singularities, Internat. J. Bifur. Chaos Appl. Sci. Engrg. 22(11), 1-20, 2012.

[10] F. Dumortier, J. Llibre and J. C. Artés, Qualitative theory of polynomial differential systems, Universitext, Springer-Verlag, 2006.

[11] X. Li, Z. Wang, Global dynamics of a cubic Hamiltonian system with a periodic annulus linked to equilibria at infinity, Math. Comput. Modelling 54, 292-300, 2011.

[12] R. D. Oliveira and A. C. Rezende, Global phase portraits of a SIS model, Appl. Math. Comput. 219(9), 4924-4930, 2013.

Departament de Matemàtiques. Edifici C. 08193 Bellaterra (Barcelona, Spain)

E-mail address: leen@mat.uab.cat, torre@mat.uab.cat 